Algebraic $\&$ Geometric Topology

Volume 3 (2003) 511-535

Published: 15 June 2003

ATG

\title{
Torsion in Milnor fiber homology
}

\author{
Daniel C. Cohen \\ Graham Denham \\ AleXANDER I. Suciu
}

\begin{abstract}
In a recent paper, Dimca and Némethi pose the problem of finding a homogeneous polynomial $f$ such that the homology of the complement of the hypersurface defined by $f$ is torsion-free, but the homology of the Milnor fiber of $f$ has torsion. We prove that this is indeed possible, and show by construction that, for each prime $p$, there is a polynomial with $p$-torsion in the homology of the Milnor fiber. The techniques make use of properties of characteristic varieties of hyperplane arrangements.
\end{abstract}

AMS Classification 32S55; 14J70, 32S22, 55N25

Keywords Milnor fibration, characteristic variety, arrangement

\section{Introduction}

Let $f:\left(\mathbb{C}^{\ell+1}, \mathbf{0}\right) \rightarrow(\mathbb{C}, 0)$ be a homogeneous polynomial. Denote by $M=$ $\mathbb{C}^{\ell+1} \backslash f^{-1}(0)$ the complement of the hypersurface defined by the vanishing of $f$, and let $F=f^{-1}(1)$ be the Milnor fiber of the bundle map $f: M \rightarrow \mathbb{C}^{*}$. In [10, Question 3.10], Dimca and Némethi ask the following.

Question Suppose the integral homology of $M$ is torsion-free. Is then the integral homology of $F$ also torsion-free?

The Milnor fiber $F$ has the homotopy type of a finite, $\ell$-dimensional CWcomplex. If $f$ has an isolated singularity at $\mathbf{0}$ (for example, if $\ell=1$ ), then $F$ is homotopic to a bouquet of $\ell$-spheres, and so $H_{*}(F ; \mathbb{Z})$ is torsion-free. The purpose of this paper is to prove the following result, which provides a negative answer to the above question, as soon as $\ell>1$.

Theorem 1 Let $p$ be a prime number, and let $\ell$ be an integer greater than 1 . Then there is a homogeneous polynomial $f_{p, \ell}: \mathbb{C}^{\ell+1} \rightarrow \mathbb{C}$ for which $H_{*}(M ; \mathbb{Z})$ is torsion-free, but $H_{1}(F ; \mathbb{Z})$ has $p$-torsion. 
Let $x_{1}, \ldots, x_{\ell+1}$ be coordinates for $\mathbb{C}^{\ell+1}$. The theorem is proven by finding criteria for the construction of such polynomials, then by explicitly exhibiting a family of 3 -variable polynomials $f_{p}=f_{p}\left(x_{1}, x_{2}, x_{3}\right)$ with the desired properties, for all primes $p$ :

$$
f_{p}= \begin{cases}x_{1} x_{2}\left(x_{1}^{p}-x_{2}^{p}\right)^{2}\left(x_{1}^{p}-x_{3}^{p}\right)\left(x_{2}^{p}-x_{3}^{p}\right), & \text { if } p \text { is odd } \\ x_{1}^{2} x_{2}\left(x_{1}^{2}-x_{2}^{2}\right)^{3}\left(x_{1}^{2}-x_{3}^{2}\right)^{2}\left(x_{2}^{2}-x_{3}^{2}\right), & \text { if } p=2 .\end{cases}
$$

It then suffices to take $f_{p, \ell}\left(x_{1}, \ldots, x_{\ell+1}\right)=f_{p}\left(x_{1}, x_{2}, x_{3}\right)$.

The above polynomials are all products of powers of linear factors, and so define multi-arrangements of hyperplanes. See [16] as a general reference on arrangements. For each prime $p$, the underlying arrangement $\mathcal{A}_{p}$ is a deletion of the arrangement associated to the complex reflection group $G(3,1, p)$, and has defining polynomial $Q\left(\mathcal{A}_{p}\right)=x_{1} x_{2}\left(x_{1}^{p}-x_{2}^{p}\right)\left(x_{1}^{p}-x_{3}^{p}\right)\left(x_{2}^{p}-x_{3}^{p}\right)$. As is well known, for any hyperplane (multi)-arrangement, the homology groups of the complement are finitely-generated and torsion-free. Thus, Theorem 1 is a consequence of the following result, which identifies more precisely the torsion in the homology of the Milnor fiber of the corresponding multi-arrangement.

Theorem 2 Let $F_{p}=f_{p}^{-1}(1)$ be the Milnor fiber of the polynomial defined in (1). Then:

$$
H_{1}\left(F_{p} ; \mathbb{Z}\right)= \begin{cases}\mathbb{Z}^{3 p+1} \oplus \mathbb{Z}_{p} \oplus T, & \text { if } p \text { is odd, } \\ \mathbb{Z}^{3 p+1} \oplus \mathbb{Z}_{2} \oplus \mathbb{Z}_{2}, & \text { if } p=2,\end{cases}
$$

where $T$ is a finite abelian group satisfying $T \otimes \mathbb{Z}_{q}=0$ for every prime $q$ such that $q \nmid 2(2 p+1)$.

The $p$-torsion in $H_{1}\left(F_{p} ; \mathbb{Z}\right)$ is the smallest it can be (without being trivial). Indeed, if $H_{*}(M ; \mathbb{Z})$ is torsion-free, then an application of the Wang sequence for the Milnor fibration $F \rightarrow M \rightarrow \mathbb{C}^{*}$ shows that if the 2-torsion summand of $H_{1}(F ; \mathbb{Z})$ is non-trivial, then it must contain a repeated factor (compare [10, Prop. 3.11]).

The complement $M$ of a (central) arrangement of $n$ hyperplanes admits a minimal cell decomposition, that is, a cell decomposition for which the number of $k$-cells equals the $k$-th Betti number, for each $k \geq 0$, see [18], [11]. On the other hand, it is not known whether the Milnor fiber of a reduced defining polynomial for the arrangement admits a minimal cell decomposition. As noted in [18], this Milnor fiber does admit a cell decomposition with $n \cdot b_{k}(U)$ cells of dimension $k$, where $U$ is the complement of the projectivized arrangement. Our results show that there exist multi-arrangements for which the Milnor fiber 
$F$ admits no minimal cell decomposition. Indeed, by the Morse inequalities, the existence of such a cell decomposition would rule out torsion in $H_{*}(F ; \mathbb{Z})$.

This paper is organized as follows. Relevant results concerning finite abelian covers, characteristic varieties, and Milnor fibrations of multi-arrangements are reviewed in Sections 2 and 3. Criteria which insure that the homology of the Milnor fiber of a multi-arrangement has torsion are established in Section 4. Multi-arrangements arising from deletions of monomial arrangements are studied in Sections 5 and 6. The proof of Theorem 2 is completed in Section 7.

Acknowledgments Some of this work was carried out while the authors attended the March 2002 Mini-Workshop "Cohomology Jumping Loci" at the Mathematisches Forschungsinstitut Oberwolfach. We thank the Institute for its hospitality, and for providing an exciting and productive mathematical environment.

This research was supported by Louisiana Board of Regents grant LEQSF(19992002)-RD-A-01 and by National Security Agency grant MDA904-00-1-0038 (D. Cohen), by a grant from NSERC of Canada (G. Denham), and by NSF grant DMS-0105342 (A. Suciu).

\section{$2 \quad$ Finite abelian covers and cohomology jumping loci}

We start by reviewing some basic facts about finite abelian covers, and how to derive information about their homology from the stratification of the character torus of the fundamental group by cohomology jumping loci. A more detailed treatment in the case of line arrangements may be found in the survey [20].

\subsection{Homology of finite abelian covers}

Let $\left(X, x_{0}\right)$ be a based, connected space with the homotopy type of a finite CWcomplex, and let $G=\pi_{1}\left(X, x_{0}\right)$ be its fundamental group. Let $Y$ be a finite, regular, abelian cover of $X$, with deck transformation group $A$. Finally, let $\mathbb{K}$ be a field, with multiplicative group of units $\mathbb{K}^{\times}$, and let $\widehat{G}=\operatorname{Hom}\left(G, \mathbb{K}^{\times}\right)$be the group of $\mathbb{K}$-valued characters of $G$.

We shall assume that $\mathbb{K}$ is algebraically closed, and that the characteristic of $\mathbb{K}$ does not divide the order of $A$. With these assumptions, finitely-generated $\mathbb{K}[A]$-modules are semisimple. Since $A$ is abelian, irreducible representations 
are one-dimensional, given by characters $\chi: A \rightarrow \mathbb{K}^{\times}$. By composing with the map $G \rightarrow A$, we obtain one-dimensional $G$-modules denoted $\mathbb{K}_{\chi}$.

The lemma below is not new, and its proof can be found in various special cases. See [12], [19], [14] in the context of 2-complexes; [5] in the context of cyclic covers of complements of arrangements; and [2] in an algebraic setting. For completeness, we will sketch a proof of the version needed here.

Lemma 2.2 Let $p: Y \rightarrow X$ be a finite, regular, abelian cover with group of deck transformations $A$, and let $\mathbb{K}$ be an algebraically closed field, with $\operatorname{char} \mathbb{K} \nmid|A|$. Then

$$
H_{*}(Y ; \mathbb{K}) \cong \bigoplus_{\chi \in \widehat{A}} H_{*}\left(X ; \mathbb{K}_{\chi}\right),
$$

where $\mathbb{K}_{\chi}$ denotes the rank one local system given by lifting a character $\chi \in$ $\widehat{A}=\operatorname{Hom}\left(A, \mathbb{K}^{\times}\right)$to a representation of $G=\pi_{1}\left(X, x_{0}\right)$. Furthermore, the direct summand indexed by a character $\chi$ is the corresponding isotypic component of $H_{*}(Y ; \mathbb{K})$ as a $\mathbb{K}[A]$-module.

Proof The Leray spectral sequence of the cover $p: Y \rightarrow X$ degenerates to give an isomorphism

$$
H_{*}(Y ; \mathbb{K}) \cong H_{*}(X ; \mathbb{K}[A]),
$$

where the action of $G$ on $\mathbb{K}[A]$ is induced by left-multiplication of $G$ on $A=$ $\pi_{1}(X) / p_{*}\left(\pi_{1}(Y)\right)$. That is, $H_{*}(Y ; \mathbb{K})$ is the homology of $C_{*}(Y) \otimes_{\mathbb{K}[G]} \mathbb{K}[A]$, a chain complex of $A$-modules under the right action of $A$. By our assumptions on $\mathbb{K}$, all $\mathbb{K}[A]$-modules are semisimple, so the group algebra of $A$ is isomorphic, as an $A$-module, to a direct sum of (one-dimensional) irreducibles: $\mathbb{K}[A] \cong$ $\bigoplus_{\chi \in \widehat{A}} \mathbb{K}_{\chi}$. This decomposition into isotypic components commutes with $\otimes_{\mathbb{K}[G]}$ and homology, yielding (2).

\subsection{Characteristic varieties}

Assume that $H_{1}(X ; \mathbb{Z})=G^{\mathrm{ab}}$ is torsion-free and non-zero, and fix an isomorphism $\alpha: G^{\mathrm{ab}} \rightarrow \mathbb{Z}^{n}$, where $n=b_{1}(X)$. Let $\mathbb{K}$ be an algebraically closed field. The isomorphism $\alpha$ identifies the character variety $\widehat{G}=\operatorname{Hom}\left(G, \mathbb{K}^{\times}\right)$with the algebraic torus $\mathbf{T}(\mathbb{K})=\left(\mathbb{K}^{\times}\right)^{n}$.

The cohomology jumping loci, or characteristic varieties, of $X$ are the subvarieties $\Sigma_{d}^{q}(X, \mathbb{K})$ of the character torus defined by

$$
\Sigma_{d}^{q}(X, \mathbb{K})=\left\{\mathbf{t}=\left(t_{1}, \ldots, t_{n}\right) \in\left(\mathbb{K}^{\times}\right)^{n} \mid \operatorname{dim}_{\mathbb{K}} H^{q}\left(X ; \mathbb{K}_{\mathbf{t}}\right) \geq d\right\},
$$


where $\mathbb{K}_{\mathbf{t}}$ denotes the rank one local system given by the composite $G \stackrel{\text { ab }}{\longrightarrow}$ $G^{\mathrm{ab}} \stackrel{\alpha}{\rightarrow} \mathbb{Z}^{n} \stackrel{\mathrm{t}}{\rightarrow} \mathbb{K}^{\times}$, and the last homomorphism sends the $j$-th basis element to $t_{j}$. For fixed $q>0$, these loci determine a (finite) stratification

$$
\left(\mathbb{K}^{\times}\right)^{n} \supseteq \Sigma_{1}^{q}(X, \mathbb{K}) \supseteq \Sigma_{2}^{q}(X, \mathbb{K}) \supseteq \cdots \supseteq \emptyset .
$$

Define the depth of a character $\mathbf{t}: G \rightarrow \mathbb{K}^{\times}$relative to this stratification by

$$
\operatorname{depth}_{X, \mathbb{K}}^{q}(\mathbf{t})=\max \left\{d \mid \mathbf{t} \in \Sigma_{d}^{q}(X ; \mathbb{K})\right\} .
$$

The varieties $\Sigma_{d}^{1}(G, \mathbb{K})$, the jumping loci for 1-dimensional cohomology of the Eilenberg-MacLane space $K(G, 1)$, are particularly accessible. Indeed, these varieties are the determinantal varieties of the Alexander matrix associated to a (finite) presentation of $G$, see for instance [14, Rem. 5.2].

Now assume that $H_{2}(X ; \mathbb{Z})$ is also torsion-free, and that the Hurewicz homomorphism $h: \pi_{2}(X) \rightarrow H_{2}(X)$ is the zero map. Then $H^{2}(X)=H^{2}(G)$, and this readily implies $\Sigma_{d}^{1}(X, \mathbb{K})=\Sigma_{d}^{1}(G, \mathbb{K})$. Thus, we may compute $\operatorname{depth}_{\mathbb{K}}(\mathbf{t}):=$ $\operatorname{depth}_{X, \mathbb{K}}^{1}(\mathbf{t})$ directly from the Alexander matrix of $G$.

\section{$2.4 \quad$ Finite cyclic covers}

Consider the case where $A=\mathbb{Z}_{N}$ is a cyclic group of order $N$. Assume the characteristic of the field $\mathbb{K}$ does not divide $N$, so that the homomorphism $\iota: \mathbb{Z}_{N} \rightarrow \mathbb{K}^{\times}$which sends a generator of $\mathbb{Z}_{N}$ to a primitive $N$-th root of unity in $\mathbb{K}$ is an injection. For a homomorphism $\lambda: G \rightarrow \mathbb{Z}_{N}$, and an integer $j>0$, define a character $\lambda^{j}: G \rightarrow \mathbb{K}^{\times}$by $\lambda^{j}(g)=\iota(\lambda(g))^{j}$.

Let $X$ be a finite CW-complex, with $H_{1}(X)$ and $H_{2}(X)$ torsion-free, and such that the Hurewicz map $h: \pi_{2}(X) \rightarrow H_{2}(X)$ is trivial. In view of the preceding discussion, Theorem 6.1 in [14] applies as follows.

Corollary 2.5 Let $p: Y \rightarrow X$ be a regular, $N$-fold cyclic cover, with classifying map $\lambda: \pi_{1}(X) \rightarrow \mathbb{Z}_{N}$. Let $\mathbb{K}$ be an algebraically closed field, with char $\mathbb{K} \nmid N$. Then

$$
\operatorname{dim}_{\mathbb{K}} H_{1}(Y ; \mathbb{K})=b_{1}(X)+\sum_{1 \neq k \mid N} \varphi(k) \operatorname{depth}_{\mathbb{K}}\left(\lambda^{N / k}\right),
$$

where $\varphi$ is the Euler totient function.

This result was first used in [14] to detect 2-torsion in the homology of certain 3 -fold covers of the complement of the deleted $\mathrm{B}_{3}$ arrangement (see $\S 7.3$ below). We will apply this result to Milnor fibrations in what follows. 


\section{Homology of the Milnor fiber of a multi-arrange- ment}

In this section, we review some facts concerning the Milnor fibration of a complex (multi)-arrangement of hyperplanes, following [5] and [9].

\section{$3.1 \quad$ Hyperplane arrangements}

Let $\mathcal{A}$ be a central arrangement of hyperplanes in $\mathbb{C}^{\ell+1}$. The union of the hyperplanes in $\mathcal{A}$ is the zero locus of a polynomial

$$
f=Q(\mathcal{A})=\prod_{H \in \mathcal{A}} \alpha_{H},
$$

where each factor $\alpha_{H}$ is a linear form with kernel $H$. Let $\mathbb{C}^{*} \rightarrow \mathbb{C}^{\ell+1} \backslash\{\mathbf{0}\} \rightarrow$ $\mathbb{C P}^{\ell}$ be the Hopf bundle, with fiber $\mathbb{C}^{*}=\mathbb{C} \backslash\{0\}$. The projection map of this (principal) bundle takes the complement of the arrangement, $M=M(\mathcal{A})=$ $\mathbb{C}^{\ell+1} \backslash f^{-1}(0)$, to the complement $U$ of the projectivization of $\mathcal{A}$ in $\mathbb{C P}^{\ell}$. The bundle splits over $U$, and so $M=U \times \mathbb{C}^{*}$.

It is well known that $U$ is homotopy equivalent to a finite $\mathrm{CW}$-complex (of dimension at most $\ell$ ), and that $H_{*}(U ; \mathbb{Z})$ is torsion-free. Furthermore, for each $k \geq 2$, the Hurewicz homomorphism $h: \pi_{k}(U) \rightarrow H_{k}(U)$ is the zero map, see [17]. Thus, the assumptions from $\S 2.4$ hold for $X=U$.

The fundamental group $\pi_{1}(M)$ is generated by meridian loops (positively oriented linking circles) about the hyperplanes of $\mathcal{A}$. The homology classes of these loops freely generate $H_{1}(M)=\mathbb{Z}^{n}$, where $n=\operatorname{deg}(f)=|\mathcal{A}|$. We shall abuse notation and denote both a meridian loop about hyperplane $H \in \mathcal{A}$, and its image in $\pi_{1}(U)$ by the same symbol, $\gamma_{H}$. Note that these meridians may be chosen so that $\prod_{H \in \mathcal{A}} \gamma_{H}$ is null-homotopic in $U$. In fact, $\pi_{1}(U) \cong \pi_{1}(M) /\left\langle\prod_{H \in \mathcal{A}} \gamma_{H}\right\rangle$, and so $H_{1}(U)=\pi_{1}(U)^{\mathrm{ab}}=\mathbb{Z}^{n-1}$.

\subsection{The Milnor fibration}

As shown by Milnor, the restriction of $f: \mathbb{C}^{\ell+1} \rightarrow \mathbb{C}$ to $M$ defines a smooth fibration $f: M \rightarrow \mathbb{C}^{*}$, with fiber $F=f^{-1}(1)$ and monodromy $h: F \rightarrow F$ given by multiplication by a primitive $n$-th root of unity in $\mathbb{C}$.

The restriction of the Hopf map to the Milnor fiber gives rise to an $n$-fold cyclic covering $F \rightarrow U$. This covering is classified by the epimorphism $\lambda: \pi_{1}(U) \rightarrow \mathbb{Z}_{n}$ that sends all meridians $\gamma_{H}$ to the same generator of $\mathbb{Z}_{n}$. See [5] for details. 
Now fix an ordering $\mathcal{A}=\left\{H_{1}, H_{2}, \ldots, H_{n}\right\}$ on the set of hyperplanes. Let $\mathbf{a}=\left(a_{1}, a_{2}, \ldots, a_{n}\right)$ be an $n$-tuple of positive integers with greatest common divisor equal to 1 . We call such an $n$-tuple a choice of multiplicities for $\mathcal{A}$. The (unreduced) polynomial

$$
f_{\mathbf{a}}=\prod_{i=1}^{n} \alpha_{H_{i}}^{a_{i}}
$$

defines a multi-arrangement $\mathcal{A}_{\mathbf{a}}=\left\{H_{1}^{(1)}, \ldots, H_{1}^{\left(a_{1}\right)}, \ldots, H_{n}^{(1)}, \ldots, H_{n}^{\left(a_{n}\right)}\right\}$. Note that $\mathcal{A}_{\mathbf{a}}$ has the same complement $M$, and projective complement $U$, as $\mathcal{A}$, for any choice of multiplicities. Let $f_{\mathbf{a}}: M \rightarrow \mathbb{C}^{*}$ be the corresponding Milnor fibration. As we shall see, the fiber $F_{\mathbf{a}}=f_{\mathbf{a}}^{-1}(1)$ does depend significantly on a.

\subsection{Homology of the Milnor fiber}

Let $N=\sum_{i=1}^{n} a_{i}$ be the degree of $f_{\mathbf{a}}$, and let $\mathbb{Z}_{N}=\left\langle g \mid g^{N}=1\right\rangle$ be the cyclic group of order $N$, with fixed generator $g$. As in the reduced case above, the restriction of the Hopf map to $F_{\mathbf{a}}$ gives rise to an $N$-fold cyclic covering $F_{\mathbf{a}} \rightarrow U$, classified by the homomorphism $\lambda_{\mathbf{a}}: \pi_{1}(U) \rightarrow \mathbb{Z}_{N}$ which sends the meridian $\gamma_{i}$ about $H_{i}$ to $g^{a_{i}}$.

For any field $\mathbb{K}$, let $\tau:\left(\mathbb{K}^{\times}\right)^{n} \rightarrow \mathbb{K}^{\times}$be the map which sends an $n$-tuple of elements to their product. Since the meridians $\gamma_{i}$ may be chosen so that $\prod_{i=1}^{n} \gamma_{i}=1$, if $\mathbf{s} \in\left(\mathbb{K}^{\times}\right)^{n}$ satisfies $\tau(\mathbf{s})=1$, then $\mathbf{s}$ gives rise to a rank one local system on $U$, compare $\S 2.3$. We abuse notation and denote this local system by $\mathbb{K}_{\mathbf{s}}$.

Suppose that $\mathbb{K}$ is algebraically closed, and char $\mathbb{K}$ does not divide $N$. Then there is a primitive $N$-th root of unity $\xi \in \mathbb{K}$. Let $\mathbf{t} \in\left(\mathbb{K}^{\times}\right)^{n}$ be the character with $t_{i}=\xi^{a_{i}}$, for $1 \leq i \leq n$. Note that $\tau(\mathbf{t})=1$. Let $h_{\mathbf{a}}: F_{\mathbf{a}} \rightarrow F_{\mathbf{a}}$ be the geometric monodromy of the Milnor fibration $f_{\mathbf{a}}: M \rightarrow \mathbb{C}^{*}$, given by multiplying coordinates in $\mathbb{C}^{\ell+1}$ by a primitive $N$-th root of unity in $\mathbb{C}$. The action of the algebraic monodromy $\left(h_{\mathbf{a}}\right)_{*}: H_{*}\left(F_{\mathbf{a}} ; \mathbb{K}\right) \rightarrow H_{*}\left(F_{\mathbf{a}} ; \mathbb{K}\right)$ coincides with that of the deck transformations of the covering $F_{\mathbf{a}} \rightarrow U$. Lemma 2.2 yields the following.

Lemma 3.4 With notation as above, we have

$$
H_{*}\left(F_{\mathbf{a}} ; \mathbb{K}\right)=\bigoplus_{k=0}^{N-1} H_{*}\left(U ; \mathbb{K}_{\mathbf{t}^{k}}\right) .
$$

Furthermore, the $k$-th summand is an eigenspace for $\left(h_{\mathbf{a}}\right)_{*}$ with eigenvalue $\xi^{k}$. 
The next lemma appeared in [9] in the complex case. For convenience, we reproduce the proof in general.

Lemma 3.5 Let $\mathbb{K}$ be an algebraically closed field, and let $\mathbf{s} \in\left(\mathbb{K}^{\times}\right)^{n}$ be an element of finite order, with $\tau(\mathbf{s})=1$. Then there exists a choice of multiplicities a for $\mathcal{A}$ so that $H_{q}\left(U ; \mathbb{K}_{\mathbf{s}}\right)$ is a monodromy eigenspace of $H_{q}\left(F_{\mathbf{a}} ; \mathbb{K}\right)$.

Proof Let $\zeta \in \mathbb{K}$ be a primitive $k$-th root of unity, where $k$ is the order of $\mathbf{s}$. Then, for each $2 \leq i \leq n$, there is an integer $1 \leq a_{i} \leq k$ such that $s_{i}=\zeta^{a_{i}}$. By choosing either $1 \leq a_{1} \leq k$ or $k+1 \leq a_{1} \leq 2 k$ suitably, we can arrange that the sum $N=\sum_{i=1}^{n} a_{i}$ is not divisible by $p=\operatorname{char} \mathbb{K}$, if $p>0$. Since $\mathbf{s}$ and $\zeta$ both have order $k$, we have $\operatorname{gcd}\left\{a_{1}, \ldots, a_{n}\right\}=1$. Since the product of the coordinates of $\mathbf{s}$ is 1 , the integer $k$ divides $N$.

By insuring $p \nmid N$, there is an element $\xi \in \mathbb{K}$ for which $\xi^{N / k}=\zeta$. By construction, $\mathbf{a}=\left(a_{1}, \ldots, a_{n}\right)$ is a choice of multiplicities for which $\mathbf{s}=\mathbf{t}^{N / k}$ in the decomposition of Lemma 3.4 , so $H_{q}\left(U ; \mathbb{K}_{\mathbf{s}}\right)$ is a direct summand of $H_{q}\left(F_{\mathbf{a}} ; \mathbb{K}\right)$.

Remark 3.6 The choice of multiplicities a in Lemma 3.5 is not unique. As above, write $s_{i}=\zeta^{a_{i}}$ for integers $a_{i}$, where $\zeta$ is a $k$-th root of unity and $1 \leq a_{i} \leq k$. Let $\mathbf{a}=\left(a_{1}, \ldots, a_{n}\right)$. Then $H_{q}\left(U ; \mathbb{K}_{\mathbf{s}}\right)$ is also a monodromy eigenspace of $F_{\mathbf{b}}$ if $\mathbf{b}=\mathbf{a}+\lambda$, for all $\lambda \in(k \mathbb{Z})^{n}$ for which satisfy $b_{i}>0$ for each $i$ and, if $p>0, p \nmid \sum_{i=1}^{n} b_{i}$.

\section{Translated tori and torsion in homology}

\subsection{Characteristic varieties of arrangements}

Let $\mathcal{A}=\left\{H_{1}, \ldots, H_{n}\right\}$ be a central arrangement in $\mathbb{C}^{\ell+1}$. Let $M$ denote its complement, and $U$ the complement of its projectivization. Then the restriction of the Hopf fibration $\mathbb{C}^{*} \rightarrow M \rightarrow U$ induces an isomorphism $\pi_{1}(U) \cong \pi_{1}(M) /\left\langle\prod_{i=1}^{n} \gamma_{i}\right\rangle$, as in the previous section. For this reason, although the rank of $\pi_{1}(U)^{\mathrm{ab}}$ is $n-1$, we shall regard the characteristic varieties of $U$ as embedded in the character torus of $\pi_{1}(M)$ :

$$
\Sigma_{d}^{q}(U, \mathbb{K})=\left\{\mathbf{t} \in \operatorname{ker} \tau \cong\left(\mathbb{K}^{\times}\right)^{n-1} \mid \operatorname{dim}_{\mathbb{K}} H^{q}\left(U ; \mathbb{K}_{\mathbf{t}}\right) \geq d\right\},
$$

(compare with (3)), where, as above, $\tau:\left(\mathbb{K}^{\times}\right)^{n} \rightarrow \mathbb{K}^{\times}$is the homomorphism given by $\tau\left(t_{1}, \ldots, t_{n}\right)=t_{1} \cdots t_{n}$. 
Proposition 4.2 For $q \geq 1$ and $d \geq 0$,

$$
\Sigma_{d}^{q}(M, \mathbb{K})=\bigcup_{j=0}^{d} \Sigma_{d-j}^{q}(U, \mathbb{K}) \cap \Sigma_{j}^{q-1}(U, \mathbb{K}) .
$$

In particular, for $\mathbf{t} \in \operatorname{ker} \tau$, we have

$$
\operatorname{depth}_{M, \mathbb{K}}^{q}(\mathbf{t})=\operatorname{depth}_{U, \mathbb{K}}^{q}(\mathbf{t})+\operatorname{depth}_{U, \mathbb{K}}^{q-1}(\mathbf{t}) .
$$

Proof Let $\mathbb{K}_{\mathbf{t}}$ be the local system on $M$ corresponding to $\mathbf{t} \in\left(\mathbb{K}^{\times}\right)^{n}$. There is an induced local system $i^{*} \mathbb{K}_{\mathbf{t}}$ on $\mathbb{C}^{*}$, with monodromy $\tau(\mathbf{t})$, where $i: \mathbb{C}^{*} \rightarrow M$ is the inclusion of the fiber in the Hopf bundle $\mathbb{C}^{*} \rightarrow M \rightarrow U$. Fix a section $s: U \rightarrow M$ of this trivial bundle, and let $s^{*} \mathbb{K}_{\mathbf{t}}$ be the induced local system on $U$. Recall that we denote this local system by $\mathbb{K}_{\mathbf{t}}$ in the case where $\tau(\mathbf{t})=1$. To prove the Proposition, it suffices to show that, for each $q \geq 1$,

$$
H^{q}\left(M ; \mathbb{K}_{\mathbf{t}}\right)= \begin{cases}0, & \text { if } \tau(\mathbf{t}) \neq 1, \\ H^{q}\left(U ; \mathbb{K}_{\mathbf{t}}\right) \oplus H^{q-1}\left(U ; \mathbb{K}_{\mathbf{t}}\right), & \text { if } \tau(\mathbf{t})=1 .\end{cases}
$$

Let $C_{\bullet}(\widetilde{M})$ and $C_{\bullet}(\widetilde{U})$ be the chain complexes of the universal covers of $M$ and $U$, viewed as modules over the group rings of $\bar{G}=\pi_{1}(M)$ and $G=$ $\pi_{1}(U)$, respectively. Then the cohomology of $M$ with coefficients in $\mathbb{K}_{\mathbf{t}}$ is (by definition) the cohomology of the complex $\bar{C}^{\bullet}=\operatorname{Hom}_{\mathbb{Z} \bar{G}}(C \bullet(\widetilde{M}), \mathbb{K})$, where the $\mathbb{Z} \bar{G}$-module structure on $\mathbb{K}$ is given by the representation $\bar{G} \stackrel{\text { ab }}{\longrightarrow} \bar{G}^{\text {ab }} \stackrel{\alpha}{\longrightarrow}$ $\mathbb{Z}^{n} \stackrel{\mathbf{t}}{\rightarrow} \mathbb{K}^{\times}$. Similarly, $H^{*}\left(U ; s^{*} \mathbb{K}_{\mathbf{t}}\right)$ is the cohomology of the complex $\mathrm{C}^{\bullet}=$ $\operatorname{Hom}_{\mathbb{Z} G}(C \bullet(\widetilde{U}), \mathbb{K})$. Denote the boundary maps of the complexes $\bar{C}^{\bullet}$ and $\mathrm{C}^{\bullet}$ by $\Delta^{\bullet}$ and $\delta^{\bullet}$, respectively.

Multiplication by $1-\tau(\mathbf{t})$ gives rise to a chain map $C^{\bullet} \rightarrow C^{\bullet}$. Since $M=U \times \mathbb{C}^{*}$ is a product, and the monodromy of the induced local system $i^{*} \mathbb{K}_{\mathbf{t}}$ on $\mathbb{C}^{*}$ is $\tau(\mathbf{t})$, the complex $\overline{\mathrm{C}}^{\bullet}$ may be realized as the mapping cone of this chain map. Explicitly, we have $\overline{\mathrm{C}}^{q}=\mathrm{C}^{q} \oplus \mathrm{C}^{q-1}$, and $\Delta^{q}: \mathrm{C}^{q} \oplus \mathrm{C}^{q-1} \rightarrow \mathrm{C}^{q+1} \oplus \mathrm{C}^{q}$ is given by

$$
\Delta^{q}(x, y)=\left(\delta^{q}(x), \delta^{q-1}(y)+(-1)^{q}(1-\tau(\mathbf{t})) \cdot x\right) .
$$

If $\tau(\mathbf{t}) \neq 1$, it is readily checked that the complex $\bar{C}^{\bullet}$ is acyclic. If $\tau(\mathbf{t})=1$, it follows immediately from the above description of the boundary map $\Delta^{\bullet}$ that $H^{q}\left(\overline{\mathrm{C}}^{\bullet}\right) \cong H^{q}\left(\mathrm{C}^{\bullet}\right) \oplus H^{q-1}\left(\mathrm{C}^{\bullet}\right)$ for each $q$.

Now let $\mathrm{d} \mathcal{A}$ be the decone of $\mathcal{A}$ with respect to one of the hyperplanes (which, after a linear change of variables, may be assumed to be a coordinate hyperplane). The complement, $M(\mathrm{~d} \mathcal{A})$, in $\mathbb{C}^{\ell}$ is diffeomorphic to the complement 
$U$ of the projectivization of $\mathcal{A}$. An isomorphism $\pi_{1}(U) \rightarrow \pi_{1}(M(\mathrm{~d} \mathcal{A}))$ is obtained by deleting the meridian corresponding to the deconing hyperplane. Let $\pi:\left(\mathbb{K}^{\times}\right)^{n} \rightarrow\left(\mathbb{K}^{\times}\right)^{n-1}$ be the map that forgets the corresponding coordinate. Then $\pi$ induces a bijection $\pi_{\sharp}: \Sigma_{d}^{q}(U, \mathbb{K}) \rightarrow \Sigma_{d}^{q}(M(\mathrm{~d} \mathcal{A}), \mathbb{K})$.

If $\mathbf{s}$ is a nontrivial character, then $H^{0}\left(U, \mathbb{K}_{\mathbf{s}}\right)=0$ and $\operatorname{depth}_{U, \mathbb{K}}^{1}(\mathbf{s})<n-1$. Consequently, as shown in [8] using properties of Fitting ideals, for $q=1$ and $d<n$, the above proposition simplifies to:

$$
\Sigma_{d}^{1}(M(\mathcal{A}), \mathbb{K})=\left\{\mathbf{t} \in\left(\mathbb{K}^{\times}\right)^{n} \mid \pi(\mathbf{t}) \in \Sigma_{d}^{1}(M(\mathrm{~d} \mathcal{A}), \mathbb{K}) \text { and } \tau(\mathbf{t})=1\right\} .
$$

Each irreducible component of $\Sigma_{d}^{q}(U, \mathbb{C})$ (resp., $\left.\Sigma_{d}^{q}(M(\mathrm{~d} \mathcal{A}), \mathbb{C})\right)$ is a torsiontranslated subtorus of the algebraic torus $\mathbf{T}(\mathbb{C})=\left(\mathbb{C}^{\times}\right)^{n}$, see [1]. That is, each component of $\Sigma_{d}^{q}(U, \mathbb{C})$ is of the form $g T$, where $T$ is a subgroup of $\mathbf{T}(\mathbb{C})$ isomorphic to a product of 0 or more copies of $\mathbb{C}^{\times}$, and $g \in \mathbf{T}(\mathbb{C})$ is of finite order. Recall that every algebraic subgroup of $\mathbf{T}(\mathbb{K})$ can be written as the product of a finite group with a subtorus [15, p. 187]. If the order of an element $g \in \mathbf{T}(\mathbb{K})$ is finite, we will denote its order by $\operatorname{ord}(g)$.

\subsection{Jumping loci and the Milnor fibration}

Write $H_{i}=\operatorname{ker}\left(\alpha_{i}\right)$ and let $f_{\mathbf{a}}=\prod_{i=1}^{n} \alpha_{i}^{a_{i}}$ be the polynomial of degree $N=$ $\sum_{i=1}^{n} a_{i}$ corresponding to a choice of multiplicities $\mathbf{a}=\left(a_{1}, \ldots, a_{n}\right)$ for $\mathcal{A}$. Recall that $F_{\mathbf{a}}$, the Milnor fiber of $f_{\mathbf{a}}: M \rightarrow \mathbb{C}^{*}$, is the regular, $N$-fold cyclic cover of $U$ classified by the homomorphism $\lambda_{\mathbf{a}}: \pi_{1}(U) \rightarrow \mathbb{Z}_{N}$ given by $\lambda_{\mathbf{a}}\left(\gamma_{i}\right)=$ $g^{a_{i}}$. Recall also that $b_{1}(U)=|\mathcal{A}|-1=n-1$. From Corollary 2.5, we obtain the following.

Theorem 4.4 Let $\mathbb{K}$ be an algebraically closed field, with char $\mathbb{K} \nmid N$. Then

$$
\operatorname{dim}_{\mathbb{K}} H_{1}\left(F_{\mathbf{a}} ; \mathbb{K}\right)=n-1+\sum_{1 \neq k \mid N} \varphi(k) \operatorname{depth}_{\mathbb{K}}\left(\lambda_{\mathbf{a}}^{N / k}\right) .
$$

\subsection{Jumping loci in different characteristics}

Our goal for the rest of this section is to show that if a translated torus $g T$ is a positive-dimensional component of a characteristic variety $\Sigma_{d}^{q}(U, \mathbb{C})$, but $T$ itself is not a component, then there exist choices of multiplicities a for which $H_{q}\left(F_{\mathbf{a}} ; \mathbb{Z}\right)$ has integer torsion (Theorem 4.11). In fact, we will describe how to choose such exponents explicitly, and give a more general criterion for the existence of torsion (Theorem 4.9). 
We start by comparing representations of the fundamental group over fields of positive characteristic with those over $\mathbb{C}$. Let $\zeta$ be a root of unity, and denote by $\mathbb{Z}[\zeta]$ the ring of cyclotomic integers.

Lemma 4.6 Let $i: \mathbb{Z}[\zeta] \rightarrow \mathbb{C}$ and $j: \mathbb{Z}[\zeta] \rightarrow \mathbb{K}$ be ring homomorphisms, and assume that $i$ is an injection. For any $\mathbf{t} \in\left(\mathbb{Z}[\zeta]^{\times}\right)^{n}$ with $\tau(\mathbf{t})=1$, let $i_{*} \mathbf{t}$ and $j_{*} \mathbf{t}$ denote the images of $\mathbf{t}$ in $\mathbf{T}(\mathbb{C})$ and $\mathbf{T}(\mathbb{K})$, respectively. Then

$$
\operatorname{dim}_{\mathbb{C}} H_{q}\left(U ; \mathbb{C}_{i_{*} \mathbf{t}}\right) \leq \operatorname{dim}_{\mathbb{K}} H_{q}\left(U ; \mathbb{K}_{j_{*} \mathbf{t}}\right) .
$$

Proof Since the character $\mathbf{t}$ satisfies $\tau(\mathbf{t})=1$, it gives rise to a homomorphism $\psi: \mathbb{Z} G \rightarrow \mathbb{Z}[\zeta]$, where $G=\pi_{1}(U)$ and $\mathbb{Z} G$ is the integral group ring. Let $K_{*}=C_{*}(\widetilde{U}) \otimes_{\psi} \mathbb{Z}[\zeta]$ denote the corresponding tensor product of the chain complex of the universal cover of $U$ with $\mathbb{Z}[\zeta]$, a chain complex of $\mathbb{Z}[\zeta]$-modules. Then the homology groups under comparison are just those of $K_{*} \otimes_{i_{*} \mathbf{t}} \mathbb{C}$ and $K_{*} \otimes_{j_{*} \mathrm{t}} \mathbb{K}$, respectively. Since the first map $i$ is flat, the inequality follows.

Lemma 4.7 Given an arrangement $\mathcal{A}$ and positive integers $q, d$, the following two statements are equivalent.

(1) The characteristic variety $\Sigma_{d}^{q}(U, \mathbb{C})$ contains an element $g$ of finite order for which the cyclic subgroup $\langle g\rangle \nsubseteq \Sigma_{d}^{q}(U, \mathbb{C})$. Moreover, there exists $h \in\langle g\rangle \backslash \Sigma_{d}^{q}(U, \mathbb{C})$ and a prime $p$ with $p \mid \operatorname{ord}(g)$ but $p \nmid \operatorname{ord}(h)$.

(2) There exist $\mathbf{s}, \mathbf{t} \in \mathbf{T}(\mathbb{C})$, a prime $p$, and integer $r \geq 1$ for which

(a) $\operatorname{depth}_{U, \mathbb{C}}^{q}(\mathbf{t})<\operatorname{depth}_{U, \mathbb{C}}^{q}(\mathbf{s})=d$;

(b) $\operatorname{ord}\left(\mathbf{s t}^{-1}\right)=p^{r}$;

(c) $p \nmid \operatorname{ord}(\mathbf{t})$.

Proof $(1) \Rightarrow(2)$ : Write $\langle g\rangle \cong \bigoplus_{i=1}^{m} \mathbb{Z} /\left(p_{i}^{r_{i}} \mathbb{Z}\right)$, where the primes $p_{1}, p_{2}, \ldots, p_{m}$ are all distinct. For each $h \in\langle g\rangle$, define an $m$-tuple $\nu(h)$ as follows: for $1 \leq i \leq m$, let $\nu(h)_{i}=a_{i}$, where the projection of $h$ to $\mathbb{Z} /\left(p_{i}^{r_{i}} \mathbb{Z}\right)$ has order $p_{i}^{a_{i}}$. Clearly $0 \leq a_{i} \leq r_{i}$.

Let $S$ consist of those elements $h \in\langle g\rangle$ for which $h \in \Sigma_{d}^{q}(U, \mathbb{C})$. Since characteristic varieties are closed under cyclotomic Galois actions, two elements $h_{1}, h_{2} \in\langle g\rangle$ of the same order are either both in $S$ or both not in $S$. By reordering the $p_{i}$ 's, our hypothesis states that there exists $h \notin S$ with $\nu(h)=\left(a_{1}, \ldots, a_{j}, 0, \ldots, 0\right)$, for some nonzero integers $a_{1}, a_{2}, \ldots, a_{j}$, where $j<m$. Choose $h \notin S$ of this form for which $j$ is minimal. Since $\mathbf{1} \in S$ and $\nu(\mathbf{1})=(0,0, \ldots, 0)$, we have $j \geq 1$. Then for some $h^{\prime} \in\langle g\rangle$ of order $p_{j}^{r_{j}-a_{j}}$, 
we have $\nu\left(h h^{\prime}\right)=\left(a_{1}, \ldots, a_{j-1}, 0,0, \ldots, 0\right)$. By minimality, $h h^{\prime} \in S$. Then the pair of $\mathbf{t}=h$ and $\mathbf{s}=h h^{\prime}$ together with $p=p_{r}, r=r_{j}-a_{j}$ satisfy the conditions (2).

$(2) \Rightarrow(1)$ : Let $g=\mathbf{s}, h=\mathbf{t}$, and $h^{\prime}=g h^{-1}$. By hypothesis, ord $\left(h h^{\prime}\right)=$ $\operatorname{ord}(h) \operatorname{ord}\left(h^{\prime}\right)$, from which it follows that $\langle g\rangle=\left\langle h h^{\prime}\right\rangle=\left\langle h, h^{\prime}\right\rangle$. In particular, $h \in\langle g\rangle$, but by (a), $h \notin \Sigma_{d}^{q}(U, \mathbb{C})$.

\subsection{Torsion jumps}

Once again, let $\mathbb{K}$ be an algebraically closed field of positive characteristic $p$.

Theorem 4.9 If $\mathcal{A}$ is an arrangement for which the characteristic variety $\Sigma_{d}^{q}(U, \mathbb{C})$ satisfies one of the equivalent conditions of Lemma 4.7, then

$$
\operatorname{dim}_{\mathbb{K}} H_{q}\left(U ; \mathbb{K}_{\mathbf{t}}\right) \geq d
$$

Proof Let $k=\operatorname{ord}(\mathbf{t})$; from condition (2), parts (b) and (c), we have ord(s) = $p^{r} k$. Let $\zeta$ be a root of unity in $\mathbb{C}$ of order $p^{r} k$, so that $\mathbf{s}, \mathbf{t} \in\left(\mathbb{Z}[\zeta]^{\times}\right)^{n}$. Let $j: \mathbb{Z}[\zeta] \rightarrow \mathbb{K}$ be given by choosing a $k$-th root of unity $j(\zeta)$ in $\mathbb{K}$. Since $\operatorname{ord}\left(\mathbf{s t}^{-1}\right)$ is a power of the characteristic of $\mathbb{K}$, we have $j_{*} g=j_{*} h$. Then

$$
\operatorname{dim}_{\mathbb{K}} H_{q}\left(U ; \mathbb{K}_{j_{*} \mathbf{t}}\right)=\operatorname{dim}_{\mathbb{K}} H_{q}\left(U ; \mathbb{K}_{j_{*} \mathbf{s}}\right) \geq d,
$$

by condition (2)(a) and Lemma 4.6.

Corollary 4.10 Suppose $\mathcal{A}$ is an arrangement for which the characteristic variety $\Sigma_{d}^{q}(U, \mathbb{C})$ satisfies the equivalent conditions of Lemma 4.7. Then there is a choice of multiplicities a for $\mathcal{A}$ for which the group $H_{q}\left(F_{\mathbf{a}} ; \mathbb{Z}\right)$ contains $p$-torsion elements.

Proof Assume that $\mathbf{t} \in \mathbf{T}(\mathbb{C})$ satisfies condition (2)(a) of Lemma 4.7. Then, since $\mathbf{t} \notin \Sigma_{d}^{q}(U, \mathbb{C})$, we have $\operatorname{dim}_{\mathbb{C}} H_{q}\left(U ; \mathbb{C}_{\mathbf{t}}\right)<\operatorname{dim}_{\mathbb{K}} H_{q}\left(U ; \mathbb{K}_{\mathbf{t}}\right)$. Lemma 3.5 implies that there exists a choice of multiplicities a for which $H_{q}\left(U ; \mathbb{K}_{\mathbf{t}}\right)$ and $H_{q}\left(U ; \mathbb{C}_{\mathbf{t}}\right)$ are monodromy eigenspaces. Using Lemmas 3.4 and 4.6 , with one of the inequalities being strict, we find that $\operatorname{dim}_{\mathbb{C}} H_{q}\left(F_{\mathbf{a}} ; \mathbb{C}\right)<\operatorname{dim}_{\mathbb{K}} H_{q}\left(F_{\mathbf{a}} ; \mathbb{K}\right)$. The result follows.

The following statement is a special case of Theorem 4.9 that applies to some specific behavior observed in characteristic varieties (see [21] and [4]). In particular, we will use it in what follows to find torsion for our family of examples. 
Theorem 4.11 Let $\mathbf{s} T$ be a component of $\Sigma_{d}^{q}(U, \mathbb{C})$, where $T$ is a subtorus of $\mathbf{T}(\mathbb{C})$ and $\mathbf{s}$ is a finite-order element in $\mathbf{T}(\mathbb{C})$. Suppose that $T \nsubseteq \Sigma_{d}^{q}(U, \mathbb{C})$. Then there exist choices of multiplicities a for $\mathcal{A}$ for which the group $H_{q}\left(F_{\mathbf{a}} ; \mathbb{Z}\right)$ has $p$-torsion, for some prime $p$ dividing ord(s).

Proof First, note that $T$ is positive-dimensional, since $\mathbf{1}$ is contained in all non-empty characteristic varieties. Since $T$ is not contained in $\Sigma_{d}^{q}(U, \mathbb{C})$, there exist infinitely many finite-order elements $h \in T$ for which $h \notin \Sigma_{d}^{q}(U, \mathbb{C})$. (In fact, for each sufficiently large integer $k$, there exist elements $h$ with $\operatorname{ord}(h)=k$ and $h \notin \Sigma_{d}^{q}(U, \mathbb{C})$.)

Choose any element $h$ as above, of order relatively prime to that of $\mathbf{s}$, and let $u=h^{r}$ for an $r$ for which $u^{\operatorname{ord}(\mathbf{s})}=h$. Let $g=\mathbf{s} u$. Then, by construction, $g$ and $h$ satisfy the first condition of Lemma 4.7. By Corollary 4.10, $H_{q}\left(F_{\mathbf{a}} ; \mathbb{Z}\right)$ has torsion of order $p$ for those a given by Lemma 3.5.

\section{Deletions of monomial arrangements}

Now we turn to a detailed study of arrangements obtained by deleting a hyperplane from a monomial arrangement. Using results from [21] and [4], we check that these arrangements satisfy the hypotheses of Theorem 4.11. Hence, there are corresponding multi-arrangements whose Milnor fibers have torsion in homology.

\subsection{Fundamental group of the complement}

Let $\mathcal{A}_{p}$ be the arrangement in $\mathbb{C}^{3}$ defined by the homogeneous polynomial $Q\left(\mathcal{A}_{p}\right)=x_{1} x_{2}\left(x_{1}^{p}-x_{2}^{p}\right)\left(x_{1}^{p}-x_{3}^{p}\right)\left(x_{2}^{p}-x_{3}^{p}\right)$. This arrangement is obtained by deleting the hyperplane $x_{3}=0$ from the complex reflection arrangement associated to the full monomial group $G(3,1, p)$.

The projection $\mathbb{C}^{3} \rightarrow \mathbb{C}^{2}$ defined by $\left(x_{1}, x_{2}, x_{3}\right) \mapsto\left(x_{1}, x_{2}\right)$ restricts to a bundle map $M\left(\mathcal{A}_{p}\right) \rightarrow M(\mathcal{B})$, where $\mathcal{B}$ is defined by $Q(\mathcal{B})=x_{1} x_{2}\left(x_{1}^{p}-x_{2}^{p}\right)$. The fiber of this bundle is the complex line with $2 p$ points removed. Thus, $\mathcal{A}_{p}$ is a fiber-type arrangement, with exponents $(1, p+1,2 p)$. Hence, the fundamental group $G\left(\mathcal{A}_{p}\right)=\pi_{1}\left(M\left(\mathcal{A}_{p}\right)\right)$ may be realized as a semidirect product

$$
G\left(\mathcal{A}_{p}\right)=\mathbf{F}_{2 p} \rtimes_{\alpha} G(\mathcal{B}),
$$


where $\mathbf{F}_{2 p}=\pi_{1}(\mathbb{C} \backslash\{2 p$ points $\})$ is free on $2 p$ generators corresponding to the hyperplanes defined by $\left(x_{1}^{p}-x_{3}^{p}\right)\left(x_{2}^{p}-x_{3}^{p}\right)$, and $G(\mathcal{B}) \cong \mathbf{F}_{p+1} \times \mathbb{Z}$ is the fundamental group of $M(\mathcal{B})$.

The monodromy $\alpha: G(\mathcal{B}) \rightarrow \operatorname{Aut}\left(\mathbb{F}_{2 p}\right)$ which defines the semidirect product structure (6) factors as $G(\mathcal{B}) \stackrel{\eta}{\rightarrow} P_{2 p} \hookrightarrow \operatorname{Aut}\left(\mathbf{F}_{2 p}\right)$, where the inclusion of the pure braid group on $2 p$ strands $P_{2 p}$ in $\operatorname{Aut}\left(\mathbf{F}_{2 p}\right)$ is given by the restriction of the Artin representation. The "braid monodromy" $\eta: G(\mathcal{B}) \rightarrow P_{2 p}$ may be determined using the techniques of [6], [7], and [3]. In fact, this map may be obtained by an appropriate modification of the calculation in $[3, \S 2.2]$ of the braid monodromy of the full monomial arrangement defined by $x_{3} Q\left(\mathcal{A}_{p}\right)$, which we now carry out.

\subsection{Braid monodromy}

Fix a primitive $p$-th root of unity $\xi \in \mathbb{C}$. Let $B_{2 p}$ be the full braid group on $2 p$ strands, and let $\sigma_{i}, 1 \leq i \leq 2 p-1$, be the standard generators. The indices of the strands correspond to the hyperplanes $H_{i 3: r}=\operatorname{ker}\left(x_{i}-\xi^{r} x_{3}\right)$ and the generators $y_{1}, \ldots, y_{2 p}$ of $\mathbf{F}_{2 p}$, as indicated below:

$$
\begin{array}{rcccccccc}
\text { strand \# } & 1 & 2 & \cdots & p & p+1 & p+2 & \cdots & 2 p \\
\text { hyperplane } & H_{13: p} & H_{13: p-1} & \cdots & H_{13: 1} & H_{23: p} & H_{23: p-1} & \cdots & H_{23: 1} \\
\text { generator } & y_{1} & y_{2} & \cdots & y_{p} & y_{p+1} & y_{p+2} & \cdots & y_{2 p}
\end{array}
$$

Define braids $\rho_{0}, \rho_{1} \in B_{2 p}$ by

$$
\rho_{0}=\sigma_{p-1} \sigma_{p-2} \cdots \sigma_{1} \quad \text { and } \quad \rho_{1}=\tau^{-1} \sigma_{1} \sigma_{3} \cdots \sigma_{2 p-3} \sigma_{2 p-1} \tau,
$$

where

$$
\tau=\left(\sigma_{2} \sigma_{4} \cdots \sigma_{2 p-2}\right)\left(\sigma_{3} \sigma_{5} \cdots \sigma_{2 p-3}\right) \cdots\left(\sigma_{p-2} \sigma_{p} \sigma_{p+2}\right)\left(\sigma_{p-1} \sigma_{p+1}\right)\left(\sigma_{p}\right),
$$

see Figure 1. The braids $\rho_{i}$ are obtained from the "monomial braids" of [3] by deleting the central strand, corresponding to the hyperplane $H_{3}=\operatorname{ker}\left(x_{3}\right)$ in the full monomial arrangement, but not in the monomial deletion. As in [3], the braid monodromy $\eta: G(\mathcal{B}) \rightarrow P_{2 p}$ may be expressed in terms of these braids, as follows.

Define pure braids $Z_{1}, Z_{2}, A_{1,2}^{(1)}, \ldots, A_{1,2}^{(p)}$ in $P_{2 p}$ by $Z_{1}=\rho_{0}^{p}, Z_{2}=\rho_{1} \rho_{0}^{p} \rho_{1}^{-1}$, and $A_{1,2}^{(r)}=\rho_{0}^{r-p} \rho_{1}^{2} \rho_{0}^{p-r}$ for $1 \leq r \leq p$. Let $\gamma_{j}$ and $\gamma_{12: r}$ be meridian loops in $M(\mathcal{B})$ about the lines $H_{j}=\operatorname{ker}\left(x_{j}\right)$ and $H_{12: r}=\operatorname{ker}\left(x_{1}-\xi^{r} x_{2}\right)$. These loops generate the fundamental group $G(\mathcal{B})$. 

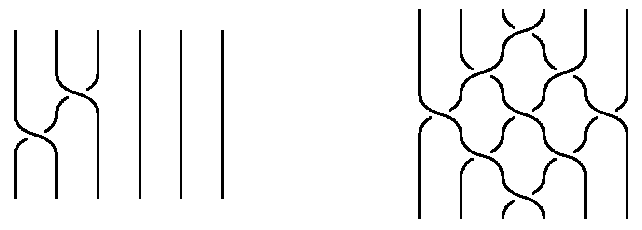

Figure 1: The braids $\rho_{0}$ and $\rho_{1}$, for $p=3$

Proposition 5.3 The braid monodromy $\eta: G(\mathcal{B}) \rightarrow P_{2 p}$ of the fiber bundle $M\left(\mathcal{A}_{p}\right) \rightarrow M(\mathcal{B})$ is given by $\eta\left(\gamma_{j}\right)=Z_{j}, \eta\left(\gamma_{12: r}\right)=A_{1,2}^{(r)}$.

Corollary 5.4 The fundamental group of $M\left(\mathcal{A}_{p}\right)$ has presentation

$$
G\left(\mathcal{A}_{p}\right)=\left\langle\begin{array}{c|c}
\gamma_{1}, \gamma_{2}, \gamma_{12: 1}, \ldots, \gamma_{12: p} & \gamma_{j}^{-1} y_{i} \gamma_{j}=\eta\left(\gamma_{j}\right)\left(y_{i}\right) \\
y_{1}, y_{2}, y_{3}, \ldots, y_{2 p} & \gamma_{12: r}^{-1} y_{i} \gamma_{12: r}=\eta\left(\gamma_{12: r}\right)\left(y_{i}\right)
\end{array}\right\rangle,
$$

where $i=1, \ldots, 2 p, j=1,2$, and $r=1, \ldots, p$, and the pure braids $\eta(\gamma)$ act on the free group $\mathbf{F}_{2 p}=\left\langle y_{1}, \ldots, y_{2 p}\right\rangle$ by the Artin representation.

\subsection{Fundamental group of the decone}

Let $\Gamma=\gamma_{1} \gamma_{12: 1} \cdots \gamma_{12: p-1} \gamma_{2} \gamma_{12: p} \in G(\mathcal{B})$. Note that $\eta(\Gamma)=A_{[2 p]}$ is the full twist on all strands. As is well known, this braid generates the center of $P_{2 p}$. It follows that $\Gamma$ is central in $G(\mathcal{B})$, so

$$
G(\mathcal{B})=\mathbf{F}_{p+1} \times \mathbb{Z}=\left\langle\gamma_{1}, \gamma_{12: 1}, \ldots, \gamma_{12: p}\right\rangle \times\langle\Gamma\rangle .
$$

To simplify calculations in $\S 6$ below, we will work with an explicit decone of the arrangement $\mathcal{A}_{p}$, as opposed to the projectization. Let $\mathrm{d} \mathcal{A}_{p}$ denote the decone of $\mathcal{A}_{p}$ with respect to the hyperplane $H_{2}=\operatorname{ker}\left(x_{2}\right)$. This is an affine arrangement in $\mathbb{C}^{2}$ (with coordinates $\left.x_{1}, x_{3}\right)$, defined by $Q\left(\mathrm{~d} \mathcal{A}_{p}\right)=$ $x_{1}\left(x_{1}^{p}-1\right)\left(x_{1}^{p}-x_{3}^{p}\right)\left(1-x_{3}^{p}\right)$. From the above discussion, we obtain the following presentation for the fundamental group of the complement of $\mathrm{d} \mathcal{A}_{p}$ :

$$
G\left(\mathrm{~d} \mathcal{A}_{p}\right)=\left\langle\begin{array}{c|c}
\gamma_{1}, \gamma_{12: 1}, \ldots, \gamma_{12: p} & \gamma_{1}^{-1} y_{i} \gamma_{1}=\eta\left(\gamma_{1}\right)\left(y_{i}\right) \\
y_{1}, y_{2}, \ldots, y_{2 p} & \gamma_{12: r}^{-1} y_{i} \gamma_{12: r}=\eta\left(\gamma_{12: r}\right)\left(y_{i}\right)
\end{array}\right\rangle
$$

where, as before, $i=1, \ldots, 2 p$ and $r=1, \ldots, p$. 


\subsection{Characteristic varieties}

Set $n=3 p+2=\left|\mathcal{A}_{p}\right|$. Denote the coordinates of the algebraic torus $\left(\mathbb{K}^{\times}\right)^{n}$ by $z_{1}, z_{2}, z_{12: 1}, \ldots, z_{12: p}, z_{13: 1}, \ldots, z_{13: p}, z_{23: 1}, \ldots, z_{23: p}$, where $z_{i}$ corresponds to the hyperplane $H_{i}=\operatorname{ker}\left(x_{i}\right)$ and $z_{i j: r}$ to the hyperplane $H_{i j: r}=\operatorname{ker}\left(x_{i}-\xi^{r} x_{j}\right)$.

The following theorem was proved for $p=2$ in [21], and for $p \geq 2$ in [4], in the case $\mathbb{K}=\mathbb{C}$. The same proofs work for an arbitrary, algebraically closed field $\mathbb{K}$.

Theorem 5.7 In addition to components of dimension 2 or higher, the variety $\Sigma_{1}^{1}\left(M\left(\mathcal{A}_{p}\right), \mathbb{K}\right)$ has 1-dimensional components $C_{1}, \ldots, C_{p-1}$, given by

$$
\bigcup_{i=1}^{p-1} C_{i}=\left\{\begin{array}{l|l}
\left(u^{p}, v^{p}, w, \ldots, w, v, \ldots, v, u, \ldots, u\right) \in\left(\mathbb{K}^{\times}\right)^{n} & \begin{array}{c}
\sum_{j=0}^{p-1} w^{j}=0 \\
\text { and } u v w=1
\end{array}
\end{array}\right\},
$$

where $C_{i}$ is obtained by setting $w$ equal to the $i$-th power of a fixed primitive $p$-th root of unity in $\mathbb{K}$.

If char $\mathbb{K}=p$, then $C_{i}$ is a subtorus of $\left(\mathbb{K}^{\times}\right)^{n}$, so passes through the origin $\mathbf{1}$. However, if char $\mathbb{K} \neq p$, then $C_{i}$ is a subtorus translated by a character of order $p$. The results of $\S 4.8$ imply that there exist choices of multiplicities a for $\mathcal{A}_{p}$ such that the first homology group of the corresponding Milnor fiber, $F_{\mathbf{a}}$, has $p$-torsion. In particular, we have the following.

Corollary 5.8 Let $F_{p}=f_{p}^{-1}(1)$ be the Milnor fiber of the polynomial defined in $(1)$. Then $H_{1}\left(F_{p} ; \mathbb{Z}\right)$ has $p$-torsion.

Proof Let $U=M\left(\mathrm{~d} \mathcal{A}_{p}\right)$ be the complement of the projectivization of $\mathcal{A}_{p}$. Note that for $\mathbf{t} \in C_{i}$, we have $\tau(\mathbf{t})=1$. So $C_{i} \subset \Sigma_{1}^{1}(U, \mathbb{K})$ by Proposition 4.2. In the case $\mathbb{K}=\mathbb{C}$, let $\mathbf{s}_{i}=\left(1,1, \xi^{i}, \ldots, \xi^{i}, \xi^{-i}, \ldots, \xi^{-i}, 1, \ldots, 1\right)$. where $\xi=$ $\exp (2 \pi \mathrm{i} / p)$, and

$$
T=\left\{\left(u^{p}, v^{p}, 1, \ldots, 1, v, \ldots, v, u, \ldots, u\right) \in\left(\mathbb{C}^{\times}\right)^{n} \mid u v=1\right\} .
$$

Then $\operatorname{ord}\left(\mathbf{s}_{i}\right)=p, T$ is a one-dimensional subtorus of $\left(\mathbb{C}^{\times}\right)^{n}$, and $C_{i}=\mathbf{s}_{i} T$. One can check that $T \nsubseteq \Sigma_{1}^{1}(U, \mathbb{C})$ using known properites of characteristic varieties of arrangements, see [13]. Hence, Theorem 4.11 implies that there are choices of multiplicities a for $\mathcal{A}_{p}$ for which $H_{1}\left(F_{\mathbf{a}} ; \mathbb{Z}\right)$ has $p$-torsion. Arguing as in the proof of that theorem, and using Lemma 3.5, reveals that among these choices of multiplicities are $\mathbf{a}=(2,1,3,3,2,2,1,1)$ in the case $p=2$, and $\mathbf{a}=(1,1,2, \ldots, 2,1, \ldots, 1,1, \ldots, 1)$ in the case $p \neq 2$. These choices yield the polynomials $f_{p}$ of (1). 


\section{Homology calculations}

Keeping the notation from the previous section, we analyze the homology of $G\left(\mathrm{~d} \mathcal{A}_{p}\right)=\pi_{1}\left(M\left(\mathrm{~d} \mathcal{A}_{p}\right)\right)$ with coefficients in the rank one local systems that arise in the study of the Milnor fibration $f_{p}: M\left(\mathcal{A}_{p}\right) \rightarrow \mathbb{C}^{*}$. In this section, we consider the case where $p \neq 2$ is an odd prime.

Let $\mathbb{K}$ be an algebraically closed field. Recall that $\mathrm{d} \mathcal{A}_{p}$ is the decone of $\mathcal{A}_{p}$ with respect to the hyperplane $H_{2}=\operatorname{ker}\left(x_{2}\right)$, which has multiplicity 1 in the multi-arrangement defined by $f_{p}$. Consequently, to analyze the homology of the Milnor fiber $F_{p}$ using Theorem 4.4, we will consider the modules $\mathbb{K}_{\mathbf{t}(k)}$ corresponding to characters $\mathbf{t}(k)$ defined by

$$
\mathbf{t}(k)=\left(t, t^{2}, \ldots, t^{2}, t, \ldots, t, t, \ldots, t\right) \in\left(\mathbb{K}^{\times}\right)^{n-1},
$$

where $t=\zeta^{N / k}$ is a power of a primitive $N$-th root of unity, $N=4 p+2, k \neq 1$ is a positive integer dividing $N$, and $n=3 p+2$.

Proposition 6.1 If $k \neq 2$ and char $\mathbb{K} \nmid N$, then $H_{1}\left(G\left(\mathrm{~d} \mathcal{A}_{p}\right) ; \mathbb{K}_{\mathbf{t}(k)}\right)=0$.

Proof The braid $Z_{1}=\eta\left(\gamma_{1}\right)$ is a full twist on strands 1 through $p$, given in terms of the standard generators $A_{i, j}$ of $P_{2 p}$ by

$$
Z_{1}=A_{1,2}\left(A_{1,3} A_{2,3}\right) \cdots \cdots\left(A_{1, p} \cdots A_{p-1, p}\right) .
$$

Consider the generating set $\left\{u_{1}, \ldots, u_{p}, v_{1}, \ldots, v_{p}\right\}$ for the free group $\mathbf{F}_{2 p}$ given by $u_{r}=y_{1} y_{2} \cdots y_{r}$ and $v_{r}=y_{p+r}, 1 \leq r \leq p$. The action of the braid $Z_{1}$ on this generating set is given by $Z_{1}\left(u_{i}\right)=u_{p} u_{i} u_{p}^{-1}$ and $Z_{1}\left(v_{j}\right)=v_{j}$ for $1 \leq i, j \leq p$, see $[7, \S 6.4]$.

Taking $\gamma_{1}, \gamma_{12: r}, u_{r}, v_{r}(1 \leq r \leq p)$ as generators for $G\left(\mathrm{~d} \mathcal{A}_{p}\right)$, we obtain from (8) a presentation with relations

$$
\begin{aligned}
u_{i} \gamma_{1} u_{p} & =\gamma_{1} u_{p} u_{i}, & u_{p} \gamma_{1} & =\gamma_{1} u_{p}, \\
u_{j} \gamma_{12: r} & =\gamma_{12: r} A_{1,2}^{(r)}\left(u_{j}\right), & v_{j} \gamma_{12: r} & =\gamma_{12: r} A_{1,2}^{(r)}\left(v_{j}\right),
\end{aligned} \quad v_{j} \gamma_{1}=\gamma_{1} v_{j},
$$

where $1 \leq i \leq p-1,1 \leq j \leq p$, and $1 \leq r \leq p$.

Let $\mathbf{A}$ denote the Alexander matrix obtained from this presentation by taking Fox derivatives and abelianizing. This is a $2 p(p+1) \times(3 p+1)$ matrix with entries in the ring of Laurent polynomials in the variables $\gamma_{1}, \gamma_{12: r}, u_{r}, v_{r}$, and 
has the form

$$
\mathbf{A}=\left(\begin{array}{cccccc}
\Delta & 0 & \cdots & 0 & 0 & \mathbb{I}_{2 p}-\gamma_{1} \Theta\left(Z_{1}\right) \\
0 & \Delta & & 0 & 0 & \mathbb{I}_{2 p}-\gamma_{12: 1} \Theta\left(A_{1,2}^{(1)}\right) \\
\vdots & & \ddots & & & \vdots \\
0 & 0 & & \Delta & 0 & \mathbb{I}_{2 p}-\gamma_{12: p-1} \Theta\left(A_{1,2}^{(p-1)}\right) \\
0 & 0 & \cdots & 0 & \Delta & \mathbb{I}_{2 p}-\gamma_{12: p} \Theta\left(A_{1,2}^{(p)}\right)
\end{array}\right),
$$

where $\Delta$ is the column vector $\left(u_{1}-1, \ldots, u_{p}-1, v_{1}-1, \ldots, v_{p}-1\right)^{\top}, \mathbb{I}_{m}$ is the $m \times m$ identity matrix, and $\Theta: P_{2 p} \rightarrow \operatorname{GL}\left(2 p, \mathbb{Z}\left[u_{1}^{ \pm 1}, \ldots, u_{p}^{ \pm 1}, v_{1}^{ \pm 1}, \ldots, v_{p}^{ \pm 1}\right]\right)$ is the Gassner representation.

Let $\mathbf{A}(k)$ denote the evaluation of the Alexander matrix at the character $\mathbf{t}(k)$. This evaluation is given by $\gamma_{1} \mapsto t, \gamma_{12: r} \mapsto t^{2}, y_{i} \mapsto t$, so $u_{r} \mapsto t^{r}$ and $v_{r} \mapsto t$. To show that $H_{1}\left(G\left(\mathrm{~d} \mathcal{A}_{p}\right) ; \mathbb{K}_{\mathbf{t}(k)}\right)=0$, it suffices to show that $\mathbf{A}(k)$ has rank $3 p$.

A calculation (compare [7, Prop. 6.6]) reveals that the evaluation at $\mathbf{t}(k)$ of $\mathbb{I}_{2 p}-\gamma_{1} \Theta\left(Z_{1}\right)$ is upper triangular, with diagonal entries $1-t^{p+1}$ and $1-t$. Recall that $\zeta$ is a primitive $N$-th root of unity, where $N=4 p+2$, that $k \neq 1$ divides $N$, and that $t=\zeta^{N / k}$. Since $p$ is prime and $k \neq 2$ by hypothesis, $k$ does not divide $p+1$. Consequently, all of the diagonal entries of the evaluation at $\mathbf{t}(k)$ of $\mathbb{I}_{2 p}-\gamma_{1} \Theta\left(Z_{1}\right)$ are nonzero. It follows that $\operatorname{rank} \mathbf{A}(k)=3 p$.

For the character $\mathbf{t}(2)=(-1,1, \ldots, 1,-1, \ldots,-1,-1, \ldots,-1)$, and the corresponding module $\mathbb{K}_{\mathbf{t}(2)}$, there are several cases to consider.

First, note that if char $\mathbb{K}=2$, then $\mathbf{t}(2)=\mathbf{1}$ is the trivial character.

If char $\mathbb{K}=p$, Theorem 5.7 and equation (5) combine to show that $\mathbf{t}(2) \in$ $\Sigma_{1}^{1}\left(M\left(\mathrm{~d} \mathcal{A}_{p}\right), \mathbb{K}\right)$. Moreover, $\mathbf{t}(2) \neq \mathbf{1}$, since $p \neq 2$. Hence, in this case the depth of $\mathbf{t}(2)$ is at least 1 .

If char $\mathbb{K} \neq 2$ or $p$, one can show that the character $\mathbf{t}(2)$ does not lie in any component of $\Sigma_{1}^{1}\left(M\left(\mathrm{~d} \mathcal{A}_{p}\right), \mathbb{K}\right)$ of positive dimension. However, this does not rule out the possibility that $\mathbf{t}(2)$ is an isolated point in $\Sigma_{1}^{1}\left(M\left(\mathrm{~d} \mathcal{A}_{p}\right), \mathbb{K}\right)$. This is not the case, as the next result shows.

Proposition 6.2 Let $\mathbb{K}$ be an algebraically closed field. If char $\mathbb{K}=p$, then $\operatorname{depth}_{\mathbb{K}}(\mathbf{t}(2))=1$. If char $\mathbb{K} \neq 2$ or $p$, then $\operatorname{depth}_{\mathbb{K}}(\mathbf{t}(2))=0$.

We will sketch a proof of this proposition by means of a sequence of lemmas. As above, we will analyze the Alexander matrix arising from a well-chosen presentation of the group $G\left(\mathrm{~d} \mathcal{A}_{p}\right)$. 
The presentation of $G\left(\mathrm{~d} \mathcal{A}_{p}\right)$ given in (8) is obtained from the realization of this group as a semidirect product, $G\left(\mathrm{~d} \mathcal{A}_{p}\right)=\mathbf{F}_{2 p} \rtimes_{\bar{\alpha}} \mathbf{F}_{p+1}$. The homomorphism $\bar{\alpha}: \mathbf{F}_{p+1} \rightarrow \operatorname{Aut}\left(\mathbb{F}_{2 p}\right)$ is the composition of the Artin representation with the braid monodromy $\bar{\eta}: \mathbf{F}_{p+1} \rightarrow P_{2 p}$ given by $\bar{\eta}: \gamma_{1} \mapsto Z_{1}, \gamma_{12: r} \mapsto A_{1,2}^{(r)}$. We first modify the map $\bar{\eta}$, as follows.

Recall the braid $\tau \in B_{2 p}$ from (7). Conjugation by $\tau$ induces an automorphism $\operatorname{cong}_{\tau}: P_{2 p} \rightarrow P_{2 p}, \beta \mapsto \tau \beta \tau^{-1}$. Then, $\operatorname{cong}_{\tau} \circ \bar{\eta}: \mathbf{F}_{p+1} \rightarrow P_{2 p}$ is another choice of braid monodromy for the (fiber-type) arrangement $\mathrm{d} \mathcal{A}_{p}$, and the presentation of $G\left(\mathrm{~d} \mathcal{A}_{p}\right)$ resulting from composing cong ${ }_{\tau} \circ \bar{\eta}$ and the Artin representation is equivalent to that obtained from $\bar{\alpha}$.

Lemma 6.3 In terms of the standard generating set for the pure braid group $P_{2 p}$, the braids $\operatorname{cong}_{\tau}\left(A_{1,2}^{(p)}\right)$ and $\operatorname{cong}_{\tau}\left(Z_{1}\right)$ are given by

$$
\begin{aligned}
\operatorname{cong}_{\tau}\left(A_{1,2}^{(p)}\right) & =A_{1,2} A_{3,4} \cdots A_{2 p-1,2 p}, \\
\operatorname{cong}_{\tau}\left(Z_{1}\right) & =A_{1,3}\left(A_{1,5} A_{3,5}\right) \cdots\left(A_{1,2 p-1} A_{3,2 p-1} \cdots A_{2 p-3,2 p-1}\right) .
\end{aligned}
$$

Proof Recall that $\rho_{0}=\sigma_{p-1} \sigma_{p-2} \cdots \sigma_{1}, \rho_{1}=\tau^{-1} \sigma_{1} \sigma_{3} \cdots \sigma_{2 p-1} \tau$, and $A_{1,2}^{(r)}=$ $\rho_{0}^{r-p} \rho_{1}^{2} \rho_{0}^{p-r}$. Hence, $\operatorname{cong}_{\tau}\left(A_{1,2}^{(p)}\right)=\sigma_{1}^{2} \sigma_{3}^{2} \cdots \sigma_{2 p-1}^{2}=A_{1,2} A_{3,4} \cdots A_{2 p-1,2 p}$. Also recall that $Z_{1}=A_{[p]}=A_{1,2}\left(A_{1,3} A_{2,3}\right) \cdots \cdots\left(A_{1, p} A_{2, p} \cdots A_{p-1, p}\right)$ is the full twist on strands 1 through $p$. We will show that $\operatorname{cong}_{\tau}\left(Z_{1}\right)$ is as asserted (for any integer $p \geq 2$ ) by induction on $p$.

Write $\tau=\tau_{p}$. When $p=2$, we have $\tau_{2} A_{[2]} \tau_{2}^{-1}=\sigma_{2} \sigma_{1}^{2} \sigma_{2}^{-1}=A_{1,3}$. So inductively assume that $\tau_{p} A_{[p]} \tau_{p}^{-1}=A_{O[p]}$, where $O[p]=\{1,3, \ldots, 2 p-1\}$. Using (7) and the braid relations, we have $\tau_{p+1}=\tau_{p} \beta_{p+1}$, where $\beta_{p+1}=\sigma_{2 p} \sigma_{2 p-1} \cdots \sigma_{p+1}$. Note that $\beta_{p+1}$ commutes with $A_{[p]}$. Hence,

$$
\begin{aligned}
\tau_{p+1} A_{[p+1]} \tau_{p+1}^{-1} & =\tau_{p} \beta_{p+1} A_{[p]}\left(A_{1, p+1} A_{2, p+1} \cdots A_{p, p+1}\right) \beta_{p+1}^{-1} \tau_{p}^{-1} \\
& =\tau_{p} A_{[p]} \tau_{p}^{-1} \cdot \tau_{p} \beta_{p+1}\left(A_{1, p+1} A_{2, p+1} \cdots A_{p, p+1}\right) \beta_{p+1}^{-1} \tau_{p}^{-1} \\
& =A_{O[p]} \cdot \tau_{p} \beta_{p+1}\left(A_{1, p+1} A_{2, p+1} \cdots A_{p, p+1}\right) \beta_{p+1}^{-1} \tau_{p}^{-1} \\
& =A_{O[p]} \cdot \tau_{p}\left(A_{1,2 p+1} A_{2,2 p+1} \cdots A_{p, 2 p+1}\right) \tau_{p}^{-1}
\end{aligned}
$$

by induction, and the readily checked fact that $\beta_{p+1} A_{i, p+1} \beta_{p+1}^{-1}=A_{i, 2 p+1}$. The result now follows from the equality $\tau_{p} A_{i, 2 p+1} \tau_{p}^{-1}=A_{2 i-1,2 p+1}$, which may itself be established by an inductive argument.

Write $\mathfrak{z}=\operatorname{cong}_{\tau}\left(Z_{1}\right)$ and $\mathfrak{a}=\operatorname{cong}_{\tau}\left(A_{1,2}^{(p)}\right)$. We specify a generating set for the free group $\mathbf{F}_{2 p}=\left\langle y_{1}, \ldots, y_{2 p}\right\rangle$ for which the action of these braids is tractable. 
For $1 \leq r \leq p$, let $u_{r}=y_{1} y_{2} \cdots y_{2 r-1} y_{2 r}$ and $v_{r}=y_{2 r-1}$. Write $V=v_{1} v_{2} \cdots v_{p}$. It is readily checked that the set of elements $\left\{u_{1}, \ldots, u_{p}, v_{1}, \ldots, v_{p}\right\}$ generates $\mathbf{F}_{2 p}$. Moreover, a calculation using the Artin representation yields the following.

Lemma 6.4 The action of the braids $\mathfrak{z}$ and $\mathfrak{a}$ on the set $\left\{u_{r}, v_{r}\right\}_{r=1}^{p}$ is given by

$$
\begin{array}{ll}
\mathfrak{z}\left(u_{r}\right)=u_{r}\left[v_{r+1} \cdots v_{p}, v_{1} \cdots v_{r}\right], & \mathfrak{a}\left(u_{r}\right)=u_{r}, \\
\mathfrak{z}\left(v_{r}\right)=V v_{r} V^{-1}, & \mathfrak{a}\left(v_{r}\right)=u_{r-1}^{-1} u_{r} v_{r} u_{r}^{-1} u_{r-1} .
\end{array}
$$

Note that $\mathfrak{z}\left(u_{p}\right)=u_{p}$ and that $\mathfrak{a}\left(v_{1}\right)=u_{1} v_{1} u_{1}^{-1}$.

Now consider the presentation of the group $G\left(\mathrm{~d} \mathcal{A}_{p}\right)$ obtained from the braid monodromy $\operatorname{cong}_{\tau} \circ \bar{\eta}: \mathbf{F}_{p+1} \rightarrow P_{2 p}$ and the Artin representation, using the generating set $\left\{u_{r}, v_{r}\right\}_{r=1}^{p}$ for the free group $\mathbf{F}_{2 p}$. Identify the generators $\gamma_{1}, \gamma_{12: j}$ of $\mathbf{F}_{p+1}$ with their images in $P_{2 p}$ via $\gamma_{1} \mapsto \operatorname{cong}_{\tau}\left(\gamma_{1}\right)=\mathfrak{z}, \gamma_{12: p} \mapsto$ $\operatorname{cong}_{\tau}\left(\gamma_{12: p}\right)=\mathfrak{a}$, and write $\gamma_{12: j} \mapsto \operatorname{cong}_{\tau}\left(\gamma_{12: j}\right)=\mathfrak{a}_{j}$ for $1 \leq j \leq p-1$. With this notation, the presentation for $G\left(\mathrm{~d} \mathcal{A}_{p}\right)$ has relations

$$
\begin{array}{ll}
u_{r} \mathfrak{z}=\mathfrak{z} u_{r}\left[v_{r+1} \cdots v_{p}, v_{1} \cdots v_{r}\right], & u_{r} \mathfrak{a}=\mathfrak{a} u_{r}, \\
v_{r} \mathfrak{z}=\mathfrak{z} V v_{r} V^{-1}, & v_{r} \mathfrak{a}=\mathfrak{a} u_{r-1}^{-1} u_{r} v_{r} u_{r}^{-1} u_{r-1},
\end{array}
$$

and $u_{r} \mathfrak{a}_{j}=\mathfrak{a}_{j} \cdot \mathfrak{a}_{j}\left(u_{r}\right), v_{r} \mathfrak{a}_{j}=\mathfrak{a}_{j} \cdot \mathfrak{a}_{j}\left(v_{r}\right)$, for $1 \leq j \leq p-1$ and $1 \leq r \leq p$.

Let $\mathbf{A}$ be the Alexander matrix obtained from this presentation, and $\mathbf{A}(2)$ the evaluation at the character $\mathbf{t}(2)$. This evaluation is given by $\gamma_{1} \mapsto-1$, $\gamma_{12: j} \mapsto 1, y_{r} \mapsto-1$, so $\mathfrak{z} \mapsto-1, \mathfrak{a} \mapsto 1, \mathfrak{a}_{j} \mapsto 1, u_{r} \mapsto 1, v_{r} \mapsto-1$. Let $\mathrm{M}$, $\mathrm{A}$, and $\mathrm{A}_{j}$ denote the evaluations at $\mathbf{t}(2)$ of the Fox Jacobians of the actions of the pure braids $\mathfrak{z}, \mathfrak{a}$, and $\mathfrak{a}_{j}$, respectively. With this notation, we have

$$
\mathbf{A}(2)=\left(\begin{array}{cccccc}
\Delta(2) & 0 & \cdots & 0 & 0 & \mathbb{I}_{2 p}+\mathrm{M} \\
0 & \Delta(2) & & 0 & 0 & \mathbb{I}_{2 p}-\mathrm{A}_{1} \\
\vdots & & \ddots & & & \vdots \\
0 & 0 & & \Delta(2) & 0 & \mathbb{I}_{2 p}-\mathrm{A}_{p-1} \\
0 & 0 & \cdots & 0 & \Delta(2) & \mathbb{I}_{2 p}-\mathrm{A}
\end{array}\right),
$$

where $\Delta(2)=\left(\begin{array}{llllll}0 & \cdots & 0 & -2 & \cdots & -2\end{array}\right)^{\top}$ is the evaluation of $\Delta$ at $\mathbf{t}(2)$. Note that the entries of $\mathbf{A}(2)$ are integers, and recall that $\mathbf{A}(2)$ has size $2 p(p+1) \times$ $(3 p+1)$.

To establish Proposition 6.2, we must show that $\operatorname{rank}_{\mathbb{K}} \mathbf{A}(2)=3 p-1$ or $3 p$, according to whether the field $\mathbb{K}$ has characteristic $p$ or not (recall that char $\mathbb{K} \neq 2$ by assumption). In the case char $\mathbb{K}=p$, we already know that $\mathbf{t}(2)$ belongs to $\Sigma_{1}^{1}\left(M\left(\mathrm{~d} \mathcal{A}_{p}\right), \mathbb{K}\right)$, so the inequality $\operatorname{rank}_{\mathbb{K}} \mathbf{A}(2) \leq 3 p-1$ holds. Thus, it suffices to prove the next result. 
Lemma 6.5 The (integral) Smith normal form of the matrix $\mathbf{A}(2)$ has diagonal entries $2, \ldots, 2$ (repeated $3 p-1$ times) and $2 p$.

Proof The matrix $\mathbf{A}(2)$ is equivalent, via row and column operations, to the matrix

$$
\left(\begin{array}{cccccc}
0 & 0 & \cdots & 0 & 0 & 2 \mathbb{I}_{2 p}+\mathrm{M}-\mathrm{A} \\
0 & \Delta(2) & & 0 & 0 & \mathbb{I}_{2 p}-\mathrm{A}_{1} \\
\vdots & & \ddots & & & \vdots \\
0 & 0 & & \Delta(2) & 0 & \mathbb{I}_{2 p}-\mathrm{A}_{p-1} \\
0 & 0 & \cdots & 0 & \Delta(2) & \mathbb{I}_{2 p}-\mathrm{A}
\end{array}\right) .
$$

A Fox calculus exercise using (10) shows that all entries of the matrices $\mathbb{I}_{2 p}-\mathrm{A}$ and $\mathbb{I}_{2 p}-\mathrm{A}_{j}, 1 \leq j \leq p-1$, are divisible by 2 , and that

$$
2 \mathbb{I}_{2 p}+\mathrm{M}-\mathrm{A}=2\left(\begin{array}{cc}
\mathbb{I}_{p} & \mathrm{P} \\
\mathrm{L}-\mathbb{I}_{p} & \mathrm{Q}
\end{array}\right)
$$

where $\mathbf{L}_{i, j}=\delta_{i, j+1}$ (Kronecker delta), $\mathbf{Q}_{i, j}=(-1)^{j+1}$, and

$$
\mathrm{P}_{i, j}= \begin{cases}(-1)^{j} & \text { if } i \text { odd and } j>i, \\ (-1)^{j+1} & \text { if } i \text { even and } j \leq i \\ 0 & \text { otherwise. }\end{cases}
$$

Let $\mathrm{U}=\left(\begin{array}{cc}\mathbb{I}_{p} & 0 \\ \mathbb{I}_{p}-\mathrm{L} & \mathbb{I}_{p}\end{array}\right)\left(\begin{array}{cc}\mathbb{I}_{p} & 0 \\ 0 & \mathrm{R}\end{array}\right)$ and $\mathrm{V}=\left(\begin{array}{cc}\mathbb{I}_{p} & -\mathrm{P} \\ 0 & \mathbb{I}_{p}\end{array}\right)\left(\begin{array}{cc}\mathbb{I}_{p} & 0 \\ 0 & \mathrm{~S}\end{array}\right)$, where

$$
\mathrm{R}=\left(\begin{array}{cccccc}
1 & 0 & 0 & \cdots & 0 & 0 \\
0 & 1 & 0 & & 0 & -1 \\
0 & 2 & 1 & & 0 & -2 \\
\vdots & & & \ddots & & \vdots \\
0 & 2 & 2 & & 1 & -(p-2) \\
0 & 2 & 2 & \cdots & 2 & -(p-2)
\end{array}\right), \quad \mathrm{S}=\left(\begin{array}{cccccc}
1 & 0 & 0 & \cdots & 0 & 0 \\
0 & 1 & 0 & & 0 & -1 \\
0 & 0 & 1 & & 0 & -2 \\
\vdots & & & \ddots & & \vdots \\
0 & 0 & 0 & & 1 & -(p-2) \\
0 & 0 & 0 & \cdots & 0 & 1
\end{array}\right) .
$$

Then one can check that $\operatorname{det} \mathrm{R}=\operatorname{det} \mathrm{S}=1$, and that $\mathrm{U}\left(2 \mathbb{I}_{2 p}+\mathrm{M}-\mathrm{A}\right) \mathrm{V}$ is a $2 p \times 2 p$ diagonal matrix with diagonal entries $2, \ldots, 2,2 p$ (in this order). Using these facts, further row and column operations reduce the matrix (11) to

$$
\left(\begin{array}{ccc}
0 & 2 \mathbb{I}_{3 p-1} & 0 \\
0 & 0 & 2 p \\
0 & 0 & \mathrm{v}
\end{array}\right)
$$

where $\mathbf{v}$ is a column vector whose entries are even integers. Now recall that if $\mathbb{K}$ is a field of characteristic $p$, then $\operatorname{rank}_{\mathbb{K}} \mathbf{A}(2) \leq 3 p-1$. Consequently, the entries of $v$ must be divisible by $p$. The result follows. 


\section{Proof of Theorem 2}

We are now in position to complete the proof of Theorem 2 from the Introduction. Recall we are given a prime $p$ and the homogenous polynomial $f_{p}$ specified in (1), and we need to compute the first homology group of the Milnor fiber $F_{p}=f_{p}^{-1}(1)$. We shall treat the cases of odd and even primes $p$ separately.

\subsection{The case $p \neq 2$}

Recall that $\mathcal{A}_{p}$ is the arrangement in $\mathbb{C}^{3}$ defined by the polynomial $Q\left(\mathcal{A}_{p}\right)=$ $x_{1} x_{2}\left(x_{1}^{p}-x_{2}^{p}\right)\left(x_{1}^{p}-x_{3}^{p}\right)\left(x_{2}^{p}-x_{3}^{p}\right)$. The choice of multiplicities

$$
\mathbf{a}=(1,1,2, \ldots, 2,1, \ldots, 1,1, \ldots, 1)
$$

yields the homogeneous polynomial $f_{p}=x_{1} x_{2}\left(x_{1}^{p}-x_{2}^{p}\right)^{2}\left(x_{1}^{p}-x_{3}^{p}\right)\left(x_{2}^{p}-x_{3}^{p}\right)$. This gives rise to a Milnor fibration $f_{p}: M\left(\mathcal{A}_{p}\right) \rightarrow \mathbb{C}^{*}$, with fiber $F_{p}=F_{\mathbf{a}}$. Let $\mathbb{Z}_{N}$ be the cyclic group of order $N=\operatorname{deg}\left(f_{p}\right)=4 p+2$, with generator $g$. The $N$-fold cyclic cover $F_{p} \rightarrow M\left(\mathrm{~d} \mathcal{A}_{p}\right)$ is classified by the epimorphism $\lambda: G\left(\mathrm{~d} \mathcal{A}_{p}\right) \rightarrow \mathbb{Z}_{N}$ given by $\lambda\left(\gamma_{1}\right)=g, \lambda\left(\gamma_{12: r}\right)=g^{2}, \lambda\left(\gamma_{i 3: r}\right)=g$.

Let $\mathbb{K}$ be an algebraically closed field, of characteristic not dividing $N$. The homology group $H_{1}\left(F_{p} ; \mathbb{K}\right)$ may be calculated using Theorem 4.4:

$$
\operatorname{dim}_{\mathbb{K}} H_{1}\left(F_{p} ; \mathbb{K}\right)=3 p+1+\sum_{1 \neq k \mid N} \varphi(k) \operatorname{depth}_{\mathbb{K}} \mathbf{t}(k),
$$

where $\mathbf{t}(k)$ are the characters defined in (9). Using Propositions 6.1 and 6.2, we find:

$$
\operatorname{dim}_{\mathbb{K}} H_{1}\left(F_{p} ; \mathbb{K}\right)= \begin{cases}3 p+1, & \text { if } \operatorname{char} \mathbb{K} \nmid 2 p(2 p+1), \\ 3 p+2, & \text { if char } \mathbb{K}=p .\end{cases}
$$

Now recall that we have an isomorphism $H_{1}\left(F_{p} ; \mathbb{Z}\right) \cong H_{1}\left(G ; \mathbb{Z}\left[\mathbb{Z}_{N}\right]\right)$ between the first homology of $F_{p}$ and that of $G=G\left(\mathrm{~d} \mathcal{A}_{p}\right)$, with coefficients in the $G$ module $\mathbb{Z}\left[\mathbb{Z}_{N}\right]$ determined by the epimorphism $\lambda: G \rightarrow \mathbb{Z}_{N}$. Let $\mathbb{Z}_{2} \subset \mathbb{Z}_{N}$ be the subgroup generated by $g^{N / 2}$, and let $\mathbb{Z}\left[\mathbb{Z}_{2}\right] \subset \mathbb{Z}\left[\mathbb{Z}_{N}\right]$ be the corresponding $G$-submodule. Denote by $J$ the kernel of the augmentation map $\epsilon: \mathbb{Z}\left[\mathbb{Z}_{2}\right] \rightarrow \mathbb{Z}$. Notice that $g^{N / 2}$ acts on $J \cong \mathbb{Z}$ by multiplication by -1 . Hence, the induced $G$-module structure on $J$ is given by the composite $G \stackrel{\alpha \circ a b}{\longrightarrow} \mathbb{Z}^{3 p+1} \stackrel{\mathbf{t}(2)}{\longrightarrow}\{ \pm 1\}$, which shows that $J$ is the integral analogue of the local system $\mathbb{K}_{\mathbf{t}(2)}$. Let $Q=\mathbb{Z}\left[\mathbb{Z}_{N}\right] / J$ be the quotient $G$-module, and consider the homology long exact sequence corresponding to the coefficient sequence $0 \rightarrow J \rightarrow \mathbb{Z}\left[\mathbb{Z}_{N}\right] \rightarrow Q \rightarrow 0$ :

$$
\cdots \rightarrow H_{2}(G ; Q) \rightarrow H_{1}(G ; J) \rightarrow H_{1}\left(G ; \mathbb{Z}\left[\mathbb{Z}_{N}\right]\right) \rightarrow H_{1}(G ; Q) \rightarrow \cdots
$$


By Lemma 6.5 , we have $H_{1}(G ; J) \cong\left(\mathbb{Z}_{2}\right)^{3 p} \oplus \mathbb{Z}_{p}$. Over an algebraically closed field $\mathbb{K}$ with char $\mathbb{K} \nmid N$, the $G$-module $Q$ decomposes as the direct sum of the modules $\mathbb{K}_{\mathbf{t}(k)}, k \neq 2$, together with the trivial module. So Proposition 6.1 implies that that $H_{1}(G ; Q)$ has no $q$-torsion, for any odd prime $q$ not dividing $2 p+1$. Note that $H_{2}(G ; Q)$ is free abelian, since the cohomological dimension of $G=\mathbf{F}_{2 p} \rtimes_{\bar{\alpha}} \mathbf{F}_{p+1}$ is 2 . Applying these observations to the long exact sequence (13) reveals that the map $H_{1}(G ; J) \rightarrow H_{1}\left(G ; \mathbb{Z}\left[\mathbb{Z}_{N}\right]\right)$ induces an isomorphism on $p$-torsion. Therefore:

$$
H_{1}\left(F_{p} ; \mathbb{Z}\right)=\mathbb{Z}^{3 p+1} \oplus \mathbb{Z}_{p} \oplus T,
$$

where $T$ is a finite abelian group such that $T \otimes \mathbb{Z}_{q}=0$ if $q \nmid 2(2 p+1)$. This finishes the proof of Theorem 2 in the case $p \neq 2$.

Remark 7.2 The $p$-torsion in (14) appears in the (-1)-eigenspace of the algebraic monodromy $h_{*}$, see Lemma 3.4. Since an automorphism of $H_{1}\left(F_{p} ; \mathbb{Z}\right)$ must preserve the $p$-torsion elements, $h_{*}$ acts on the $\mathbb{Z}_{p}$ direct summand by $x \mapsto-x$.

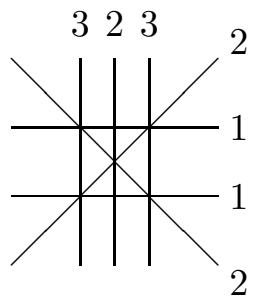

Figure 2: Decone of deleted $\mathrm{B}_{3}$ arrangement, with multiplicities

\subsection{The case $p=2$}

Now consider the arrangement $\mathcal{A}_{2}$ in $\mathbb{C}^{3}$ defined by the polynomial $Q\left(\mathcal{A}_{2}\right)=$ $x_{1} x_{2}\left(x_{1}^{2}-x_{2}^{2}\right)\left(x_{1}^{2}-x_{3}^{2}\right)\left(x_{2}^{2}-x_{3}^{2}\right)$. This is a deletion of the $\mathrm{B}_{3}$ reflection arrangement, and appears as Example 4.1 in [21] and Example 9.3 in [14]. The polynomial $f_{2}=x_{1}^{2} x_{2}\left(x_{1}^{2}-x_{2}^{2}\right)^{3}\left(x_{1}^{2}-x_{3}^{2}\right)^{2}\left(x_{2}-x_{3}\right)$ corresponds to the choice of multiplicities $\mathbf{a}=(2,1,3,3,2,2,1,1)$, shown in Figure 2 (the hyperplane at infinity has multiplicity 1$)$.

The variety $\Sigma_{1}^{1}\left(M\left(\mathcal{A}_{2}\right), \mathbb{C}\right)$ contains a 1 -dimensional component $\mathbf{s} T$, where $T=\left\{\left(u^{2}, v^{2}, 1,1, v, v, u, u\right) \mid u v=1\right\}$ and $\mathbf{s}=(1,1,-1,-1,-1,-1,1,1)$, see Theorem 5.7. The subtorus $T$ is not a component. For example, the point $\mathbf{t} \in T$ given by $u=\exp (2 \pi \mathrm{i} / 3)$ and $v=u^{2}$ is not in $\Sigma_{1}^{1}\left(M\left(\mathcal{A}_{2}\right), \mathbb{C}\right)$. 
The Milnor fiber $F_{2}=f_{2}^{-1}(1)$ is an $N$-fold cover of $M\left(\mathrm{~d} \mathcal{A}_{2}\right)$, with $N=15$. Using Theorem 4.4 as before, we find that $\operatorname{dim}_{\mathbb{K}} H_{1}\left(F_{2} ; \mathbb{K}\right)=7$ if char $\mathbb{K} \neq 2,3$, or 5 , and $\operatorname{dim}_{\mathbb{K}} H_{1}\left(F_{2} ; \mathbb{K}\right)=9$ if char $\mathbb{K}=2$. Direct computation with the Alexander matrix of $G\left(\mathrm{~d} \mathcal{A}_{2}\right)$ (see [21, Ex. 4.1]) gives the precise answer:

$$
H_{1}\left(F_{2} ; \mathbb{Z}\right)=\mathbb{Z}^{7} \oplus \mathbb{Z}_{2} \oplus \mathbb{Z}_{2} .
$$

This finishes the proof of Theorem 2 in the remaining case $p=2$.

Remark 7.4 Once again, the monodromy action preserves the torsion part in (15), so $\mathbb{Z}_{15}$ acts on $\mathbb{Z}_{2} \oplus \mathbb{Z}_{2}$. Since the torsion in $H_{1}\left(F_{2} ; \mathbb{Z}\right)$ appears in the eigenspaces of order 3 , the monodromy acts via an automorphism of order 3 , which, in a suitable basis, has matrix $\left(\begin{array}{ll}0 & 1 \\ 1 & 1\end{array}\right)$.

\section{References}

[1] D. Arapura, Geometry of cohomology support loci for local systems. I, J. Algebraic Geom. 6 (1997), no. 3, 563-597. MR 98m:32043

[2] T. Berry, Infinitesimal deformations of cyclic covers, Acta Cient. Venezolana 35 (1984), no. 3-4, 177-183. MR 86d:14011

[3] D. Cohen, Monodromy of fiber-type arrangements and orbit configuration spaces, Forum Math. 13 (2001), no. 4, 505-530. MR 2002i:52016

[4] D. Cohen, Triples of arrangements and local systems, Proc. Amer. Math. Soc. 130 (2002), 3025-3031. MR 2003g:32044

[5] D. Cohen, A. Suciu, On Milnor fibrations of arrangements, J. London Math. Soc. (2) 51 (1995), no. 1, 105-119. MR 96e:32034

[6] D. Cohen, A. Suciu, The braid monodromy of plane algebraic curves and hyperplane arrangements, Comment. Math. Helvetici 72 (1997), no. 2, 285-315. MR 98f:52012

[7] D. Cohen, A. Suciu, Homology of iterated semidirect products of free groups, J. Pure Appl. Algebra 126 (1998), no. 1-3, 87-120. MR 99e:20064

[8] D. Cohen, A. Suciu, Characteristic varieties of arrangements, Math. Proc. Cambridge Phil. Soc. 127 (1999), no. 1, 33-53. MR 2000m:32036

[9] G. Denham, The Orlik-Solomon complex and Milnor fibre homology, Topology Appl. 118 (2002), no. 1-2, 45-63. MR 2002k:32048

[10] A. Dimca, A. Némethi, Hypersurface complements, Alexander modules and monodromy, math.AG/0201291, Proceedings of the 7th Workshop on Real and Complex Singularities (Sao Carlos, 2002), to appear.

[11] A. Dimca, S. Papadima, Hypersurface complements, Milnor fibers and minimality of arrangements, math.AT/0101246, Ann. of Math., to appear. 
[12] A. Libgober, On the homology of finite abelian coverings, Topology Appl. 43 (1992), no. 2, 157-166. MR 93e:57003

[13] A. Libgober, S. Yuzvinsky, Cohomology of the Orlik-Solomon algebras and local systems, Compositio Math. 21 (2000), 337-361. MR 2001j:52032

[14] D. Matei, A. Suciu, Hall invariants, homology of subgroups, and characteristic varieties, Int. Math. Res. Not. 2002, no. 9, 465-503. MR 2003d:20055

[15] D. Northcott, Affine sets and affine groups, Cambridge University Press, Cambridge, 1980. MR 82c:14002

[16] P. Orlik, H. Terao, Arrangements of Hyperplanes, Grundlehren Math. Wiss., vol. 300, Springer-Verlag, Berlin, 1992. MR 94e:52014

[17] R. Randell, Homotopy and group cohomology of arrangements, Topology Appl. 78 (1997), no. 3, 201-213. MR 98f:52014

[18] R. Randell, Morse theory, Milnor fibers and minimality of hyperplane arrangements, Proc. Amer. Math. Soc. 130 (2002), no. 9, 2737-2743. MR 2003e:32048

[19] M. Sakuma, Homology of abelian coverings of links and spatial graphs, Canad. J. Math. 47 (1995), no. 1, 201-224. MR 96d:57008

[20] A. Suciu, Fundamental groups of line arrangements: Enumerative aspects, in: Advances in algebraic geometry motivated by physics, Contemporary Math., vol. 276, Amer. Math. Soc, Providence, RI, 2001, pp. 43-79. MR 2002k:14029

[21] A. Suciu, Translated tori in the characteristic varieties of complex hyperplane arrangements, Topology Appl. 118 (2002), no. 1-2, 209-223. MR 2002j:32027

Department of Mathematics, Louisiana State University

Baton Rouge, LA 70803, USA

Department of Mathematics, University of Western Ontario

London, ON N6A 5B7, Canada

Department of Mathematics, Northeastern University, Boston, MA 02115, USA

Email: cohen@math.lsu.edu, gdenham@uwo.ca, a.suciu@neu.edu

URL: http://www.math.lsu.edu/ ${ }^{\sim}$ cohen, http://www.math.uwo.ca/ ${ }^{\sim}$ gdenham, http://www.math.neu.edu/ suciu

Received: 14 February 2003 